\title{
A micro-accelerometer MDO benchmark problem
}

\author{
S. Tosserams · L. F. P. Etman · J. E. Rooda
}

Received: 25 March 2009 / Revised: 30 June 2009 / Accepted: 5 July 2009 / Published online: 25 September 2009

(C) The Author(s) 2009. This article is published with open access at Springerlink.com

\begin{abstract}
Many optimization and coordination methods for multidisciplinary design optimization (MDO) have been proposed in the last three decades. Suitable MDO benchmark problems for testing and comparing these methods are few however. This article presents a new MDO benchmark problem based on the design optimization of an ADXL150 type lateral capacitive micro-accelerometer. The behavioral models describe structural and dynamic effects, as well as electrostatic and amplification circuit contributions. Models for important performance indicators such as sensitivity, range, noise, and footprint area are presented. Geometric and functional constraints are included in these models to enforce proper functioning of the device. The developed models are analytical, and therefore highly suitable for benchmark and educational purposes. Four different problem decompositions are suggested for four design cases, each of which can be used for testing MDO coordination algorithms. As a reference, results for an all-in-one implementation, and a number of augmented Lagrangian coordination algorithms are given.
\end{abstract}

Keywords Multidisciplinary design optimization • Distributed optimization - Decomposition • Coordination $\cdot$ Benchmark $\cdot$ MEMS

This work is funded by MicroNed, grant number 10005898 .

S. Tosserams $(\varangle)$ · L. F. P. Etman · J. E. Rooda

Department of Mechanical Engineering,

Eindhoven University of Technology, PO Box 513,

5600 MB Eindhoven, The Netherlands

e-mail: s.tosserams@tue.nl

\section{Introduction}

Multidisciplinary design optimization (MDO) problems are encountered in the design of engineering systems that incorporate a number of interacting disciplines. Each discipline typically represents a design team concerned with the design of one aspect or component of the complete system. We use the word discipline to denote the smallest decision-making element within a system, being either a discipline in the classic MDO sense, or a component of an object-based decomposition. The goal of MDO coordination algorithms is to drive the individual disciplines towards a system design that is optimal as a whole, while maintaining some degree of design autonomy at the discipline level.

In general, MDO coordination algorithms can be divided into two classes: single-level and multilevel methods (Balling and Sobieszczanski-Sobieski 1996). Single-level methods have a centralized optimization algorithm while the analyses can be performed autonomously by the disciplines. Single-level methods allow analysis autonomy, but decision-making is centralized. The review of Cramer et al. (1994) presents an overview of single-level formulations including multidisciplinary feasible (MDF), individual discipline feasible (IDF), and all-at-once (AAO).

For multi-level methods, design optimization is distributed over the various disciplines. Each discipline is granted a degree of design freedom, and a coordination algorithm is superimposed to address the interactions between the disciplinary design problems. Well-known multi-level formulations include collaborative optimization (Braun 1996), analytical target cascading (Michelena et al. 1999), bi-level integrated system synthesis (Sobieszczanski-Sobieski et al. 2003), 
the quasiseparable decomposition method (Haftka and Watson 2005), the inexact penalty decomposition method (DeMiguel and Murray 2006), and augmented Lagrangian coordination (Tosserams et al. 2008).

Determining which coordination methods are most suitable for solving a given problem, or determining the properties of a newly proposed method, requires a comparison of both the theoretical and numerical aspects. A number of theoretical classifications are available from the engineering literature (Cramer et al. 1994; Balling and Sobieszczanski-Sobieski 1996; Alexandrov and Lewis 1999; Tosserams et al. 2009b), which focus on coordination algorithms (single-level vs. multi-level, nested vs. alternating), and the formulation of the associated optimization problems (MDF vs. IDF vs. AAO, open constraints vs. closed constraints).

Numerical comparison studies of coordination methods are available (Balling and Wilkinson 1997; Perez et al. 2004; Yi et al. 2008), but comparing and extrapolating their findings is very difficult for a number of reasons. Firstly, no unified collection of test problems is used. An effort to filling this void are the examples from the NASA MDO group (Padula et al. 1996), currently maintained by the group of prof. Bloebaum (University of Buffalo 2009). Second, reproducing realistic test problems may be hindered due to the unavailability of analysis models and/or implementation details. This is e.g. the case for the portal frame example of Sobieszczanski-Sobieski et al. (1983) and the supersonic business jet case (Agte et al. 1999). Third, test problems that can be reproduced are typically of an academic nature making extrapolation of their results to more practical design problems difficult. In general, a larger suite of representative and reproducible benchmark problems is necessary for test and comparison of coordination methods.

This article presents a new benchmark problem that can be used for test purposes in the context of coordination algorithms for decomposition-based optimal design. Although the analysis models are analytical, the design problem is representative since it captures the major considerations relevant and results in realistic designs. The Matlab analysis models are made publicly available at http://se.wtb.tue.nl/sewiki/mdo to assure the reproducibility and the comparability of results.

The benchmark problem deals with finding relevant parameters for a lateral capacitive micro-accelerometer used in e.g. airbag systems to detect decelerations in case of a collision. The design problem is based on the ADXL150 accelerometer of Analog Devices (Samuels 1996; Analog Devices Inc. 1998). Analysis models are presented for design aspects of mechanics, electrostatics, dynamics, and electronics. Most analysis models are derived from existing literature such as Zhou (1998); Mukherjee et al. (1999); Senturia (2001). The models are analytical and reproducible, and can therefore easily be used for benchmarking of optimization methods. More accurate numerical analysis routines often have the drawback of introducing numerical noise (e.g. due to the finite accuracy of finite element models), an aspect that may complicate the optimization process. Although the models are analytical, they reflect the important design considerations and are representative of the interactions present between the various disciplines.

Four design cases are developed, all of which are non-convex optimization problems. The smallest case has seven variables, and the largest has 22 variables of which two are of a discrete nature. Numerical experiments with an all-in-one implementation were performed to obtain benchmark solutions for each case. Four problem decompositions are presented that can be used for testing MDO coordination methods. Reference computational results are presented as well, generated using algorithms from the augmented Lagrangian class of coordination algorithms.

The benchmark problem was solved with an actual distributed optimization approach, thereby demonstrating that coordination methods can be used for the multidisciplinary design of microsystems. Single-level optimization of microsystem devices has been applied to microaccelerometers (Pedersen and Seshia 2004), microgyroscopes (Yuan et al. 2006), microresonators (Mukherjee et al. 1998), and microphones (Papila et al. 2006). These applications have focussed on the electromechanical optimization of material layout. Inclusion of conditioning circuit details, multiple physics, and manufacturing considerations is not straightforward, and a systematic, multi-level approach is desired according to Senturia (2001); Mukherjee (2003); Leondes (2006). MDO coordination methods have originally been developed to address similar challenges in the macro world, and may therefore also be useful in the context of multidisciplinary microsystem design.

\section{Micro-accelerometer}

We consider a lateral capacitive micro-accelerometer, similar to the ADXL150 device from Analog Devices (Samuels 1996; Analog Devices Inc. 1998). These micro-accelerometers are for example used in airbag systems. A schematic layout of the mechanical sensing element of the accelerometer is depicted in Fig. 1. The device consists of a proof mass suspended by two Usprings on each side. The U-springs are only connected 


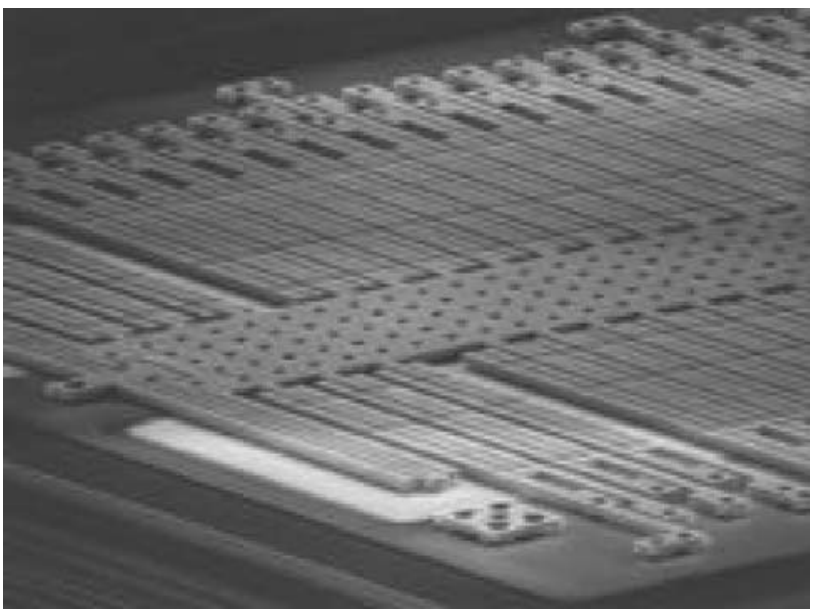

(a) Scanning electron microscope image of suspended proof mass.

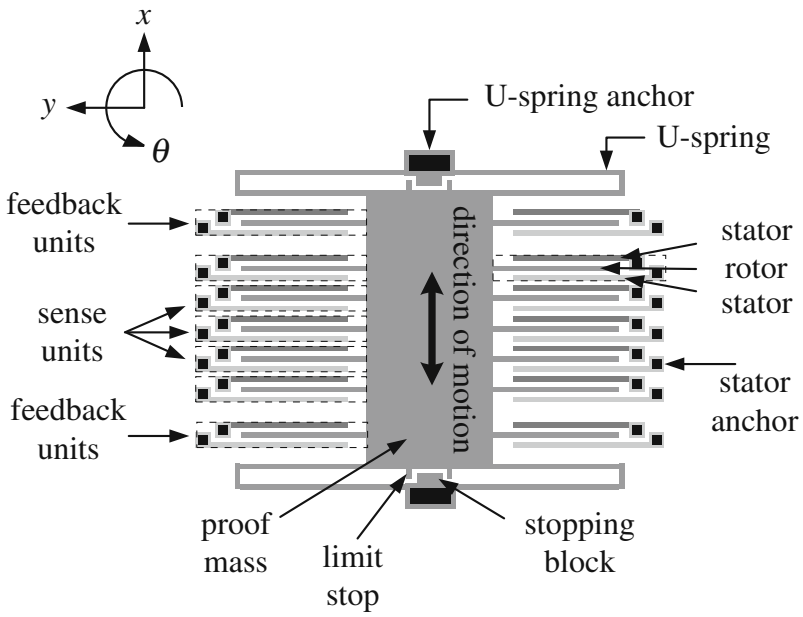

(b) Schematic illustration of the sensing mechanism (top view). Similarly shaded fingers are connected in parallel such that the total capacitance is equal to the sum of the contributions of each cell.

Fig. 1 ADXL accelerometer of Analog Devices (Samuels 1996; Analog Devices Inc. 1998). The suspended proof with comb fingers takes up an area of about $600 \mu \mathrm{m} \times 700 \mu \mathrm{m}=0.42 \mathrm{~mm}^{2}$

to the substrate at the two anchor points. A number of cantilever electrodes, called rotor fingers, are attached to the proof mass. In each sense unit, a rotor finger is positioned between two stator fingers, thereby forming a differential capacitor. The force feedback region has a similar arrangement of electrodes, but here an external voltage can be applied to introduce an electrostatic force that displaces the proof mass for self-test purposes.

When the device is exposed to an external acceleration in the sense direction, the proof mass deflects the U-springs due to inertial forces and moves relative to the substrate. The U-springs are typically flexible in one direction (the sense direction), and much stiffer in the other directions. The rotor electrodes follow the displacement of the proof mass, and cause a change in the capacitance of the sense units. This capacitance change generates a voltage, which is amplified and conditioned by the conditioning circuit.

At the two ends of the proof mass, four limit stops and two stopping blocks are used to limit the displacements of the proof mass in the $x$ and $y$ directions so that the rotor and stator fingers do not come into contact. Contact of the fingers would lead to a short circuit or possible structural damage, which may both damage the device.

Important performance measures for accelerometers include footprint area of the sensing element, and the sensitivity of the output signal with respect to an applied acceleration. Other factors to be considered during design include noise, sensing range, resonance frequencies, operational bandwidth, dynamic mode decoupling, and fabrication limitations.

\section{Analysis disciplines}

The analysis models for the accelerometer are partitioned into the four disciplines that contribute to the functionality of the device. The four subsystems are: Structures, Electrostatics, Dynamics, and Circuit. The four disciplines are coupled in two ways: The models of the subsystems may depend on the same design parameters, or the models of one subsystem depend on the outputs of another subsystem. An example of the former is that the models for Structures and Electrostatics both depend on the comb dimensions. In the latter case, Structures for example computes the responses required as inputs for the lumped model of the Dynamics subsystem. An overview of the four disciplines and their interactions is given in Fig. 2.

\subsection{Structures}

For subsystem Structures, we present analytical models for the effective mass, spring constants, and the damping coefficient as functions of the dimensions and material parameters of the device (see Fig. 3 for a definition of the dimensions). Furthermore, Structures 


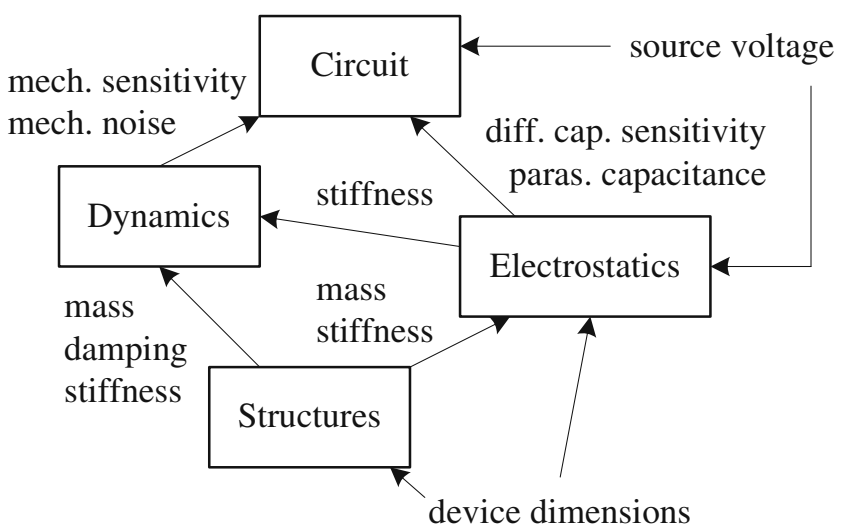

Fig. 2 Analysis disciplines and their interdependencies

includes several geometric and manufacturing design constraints that limit feature sizes, total device area, and prevent buckling. The analysis models are obtained from Mukherjee et al. (1999) and Zhou (1998), except for the mechanical spring stiffness analysis and some extensions to the damping coefficient model. The spring model is developed here from linear beam theory, and the damping model is extended to also account for the small aspect ratios in squeezed film damping using the models of Veijola et al. (2005).

\subsubsection{Area, mass, and moment of inertia}

The area of the smallest rectangle in which the accelerometer is enclosed determines the number that can be fabricated on a single wafer. Since cost benefits can be expected for smaller designs, the footprint area of the rectangle is a factor in the design. The width of this rectangle is determined by either the U-spring length or the width measured at the stator fingers, such that the area $A$ of the enclosing rectangle is

$$
A=l w=l \max \left(\left[2 l_{\mathrm{b} 1}+w_{\mathrm{a}}\right],\left[w_{\mathrm{p}}+2\left(l_{\mathrm{f}}+l_{\mathrm{s} 2}+w_{\mathrm{sa}}\right)\right]\right)
$$

where $l=l_{\mathrm{p}}+2 l_{\mathrm{b} 2}+2 l_{\mathrm{a}}$ is the total length of the design in $x$ direction, $w$ the width of the design in $y$ direction, $l_{\mathrm{p}}$ and $w_{\mathrm{p}}$ the length and width of the proof mass, $l_{\mathrm{b} 1}$ and $l_{\mathrm{b} 2}$ the lengths of beams 1 and 2 of the U-spring, $l_{\mathrm{a}}$ the length of the spring anchor, $l_{\mathrm{f}}$ the length of a rotor finger, $l_{\mathrm{s} 2}$ the additional length of the long stator finger, and $w_{\mathrm{sa}}$ the width of the stator finger anchor. Note that the thickness of the $\mathrm{U}$-spring beams in $x$ direction is neglected.

The effective moving mass $m$ can be found by a summation of the mass of the rotor fingers of the sense and feedback units, and the proof mass itself. We neglect the mass of the limit stops and the U-springs since their contributions are small. The effective mass $m$ is given by

$m=\rho h l_{\mathrm{p}} w_{\mathrm{p}}+\rho h l_{\mathrm{f}} w_{\mathrm{f}}\left(N_{\mathrm{s}}+N_{\mathrm{f}}\right)$

where each sense or feedback unit contains one rotor finger. Here $\rho=2331 \mathrm{~kg} / \mathrm{m}^{2}$ is the material density of the polysilicon fabrication material, $h$ is the structural out-of-plane height, $w_{\mathrm{f}}$ is the width of the rotor fingers, and $N_{\mathrm{s}}$ and $N_{\mathrm{f}}$ are the number of sense and feedback cells. The moment of inertia $J$ around the center of mass can be determined by a summation of the contributions of the proof mass $J_{\mathrm{p}}$, and the sense and feedback rotor fingers $J_{\mathrm{s}}$ and $J_{\mathrm{f}}$, respectively

$J=J_{\mathrm{p}}+J_{\mathrm{s}}+J_{\mathrm{f}}$

where the individual contributions are given by

$$
\begin{aligned}
& J_{\mathrm{p}}=\rho h l_{\mathrm{p}} w_{\mathrm{p}} \frac{l_{\mathrm{p}}^{2}+w_{\mathrm{p}}^{2}}{12} \\
& J_{\mathrm{s}}=2 \rho h l_{\mathrm{f}} w_{\mathrm{f}} \sum_{i=1}^{N_{\mathrm{s}} / 2}\left[\frac{l_{\mathrm{f}}^{2}+w_{\mathrm{f}}^{2}}{12}+\left(r_{\mathrm{xs}, i}^{2}+r_{\mathrm{y}}^{2}\right)\right] \\
& J_{\mathrm{f}}=4 \rho h l_{\mathrm{f}} w_{\mathrm{f}} \sum_{i=1}^{N_{\mathrm{f}} / 4}\left[\frac{l_{\mathrm{f}}^{2}+w_{\mathrm{f}}^{2}}{12}+\left(r_{\mathrm{xf}, i}^{2}+r_{\mathrm{y}}^{2}\right)\right]
\end{aligned}
$$

Due to symmetry, the number of sense units $N_{\mathrm{s}}$ is always even, and the number of feedback units $N_{\mathrm{f}}$ is a multiple of four. Here $\left(r_{\mathrm{xs}, i}, r_{\mathrm{y}}\right)$ and $\left(r_{\mathrm{xf}, i}, r_{\mathrm{y}}\right)$ are

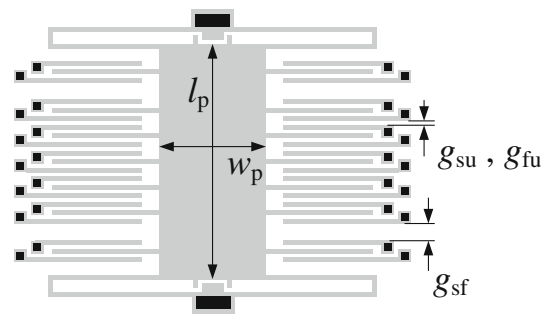

(a) Proof mass

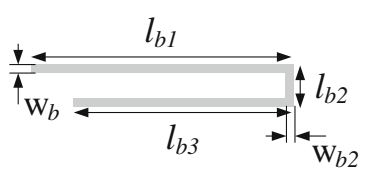

(b) U-spring

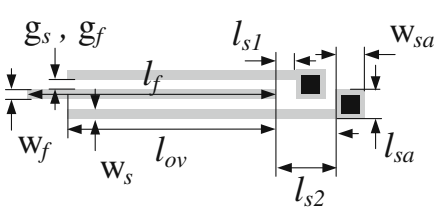

(c) Sense/feedback unit

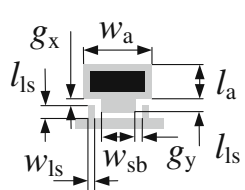

(d) Spring anchor

Fig. 3 Dimensional variables of accelerometer layout 
the coordinates of the center of mass of the $i$-th rotor finger relative to the center of the proof mass. These coordinates are given by

$$
\begin{aligned}
r_{\mathrm{xs}, i}= & l_{\mathrm{su}} / 2-w_{\mathrm{s}}-g_{\mathrm{s}}-w_{\mathrm{f}} / 2 \\
& -(i-1)\left(2 g_{\mathrm{s}}+w_{\mathrm{f}}+2 w_{\mathrm{s}}+g_{\mathrm{su}}\right) \\
r_{\mathrm{xf}, i}= & l_{\mathrm{su}} / 2+g_{\mathrm{sf}}+w_{\mathrm{s}}+g_{\mathrm{f}}+w_{\mathrm{f}} / 2 \\
& +(i-1)\left(2 g_{\mathrm{f}}+w_{\mathrm{f}}+2 w_{\mathrm{s}}+g_{\mathrm{fu}}\right) \\
r_{\mathrm{y}}= & w_{\mathrm{p}} / 2+l_{\mathrm{f}} / 2
\end{aligned}
$$

where $w_{\mathrm{s}}$ is the width of the stator fingers, $g_{\mathrm{s}}$ and $g_{\mathrm{f}}$ are the gap sizes between the sense and feedback electrodes, $g_{\mathrm{su}}$ and $g_{\mathrm{fu}}$ are the gaps between the individual sense and feedback cells, $g_{\text {sf }}$ is the gap between the sense and feedback regions, and $l_{\mathrm{su}}=N_{\mathrm{s}} / 2\left(2 g_{\mathrm{s}}+w_{\mathrm{f}}+\right.$ $\left.2 w_{\mathrm{s}}\right)+\left(N_{\mathrm{s}} / 2-1\right) g_{\mathrm{su}}$ is the total length in $x$-direction of all sense units on one side of the proof mass. Again we neglect the small contributions of the limit stops and $\mathrm{U}$ springs.

\subsubsection{Damping coefficient}

In the lumped parameter model we only consider damping in the sensing direction. Although damping also occurs in the other two directions, their contributions to the overall sensing performance are small and are therefore neglected. As a damping model, we consider two contributions: Damping due to Couette flow beneath the proof mass as the shuttle displaces $\left(b_{c}\right)$, and squeezed film damping between the fingers $\left(b_{\mathrm{s}}\right)$. The total damping coefficient $b$ is then given by $b=b_{\mathrm{c}}+b_{\mathrm{s}}$. Since the movement of the proof mass is expected to be at frequencies well below its resonance frequency, Stokes flow contributions can be neglected (Cho et al. 1994).

The damping coefficient $b_{c}$ due to Couette flow beneath the proof mass and fingers is given by (Senturia 2001)

$b_{\mathrm{c}}=\frac{\mu\left[l_{\mathrm{p}} w_{\mathrm{p}}+\left(N_{\mathrm{s}}+N_{\mathrm{f}}\right) l_{\mathrm{f}} w_{\mathrm{f}}\right]}{d}$

Fig. 4 Model for computing mechanical stiffness where $\mu=18 \cdot 10^{-6} \mathrm{Ns} / \mathrm{m}^{2}$ is the viscosity of air, and $d$ is the air gap between the proof mass and the substrate.

Squeezed film damping between the rotor and stator comb fingers (2 times $N_{\mathrm{f}}+N_{\mathrm{s}}$ gaps) can be approximated by a damping coefficient $b_{\mathrm{s}}$ (Veijola et al. 2005)

$b_{\mathrm{s}}=2 l_{\mathrm{ov}}\left[N_{\mathrm{f}} \mu_{\mathrm{e}, \mathrm{s}}\left(\frac{h+h_{\mathrm{s}}}{g_{\mathrm{s}}}\right)^{3}+N_{\mathrm{s}} \mu_{\mathrm{e}, \mathrm{f}}\left(\frac{h+h_{\mathrm{f}}}{g_{\mathrm{f}}}\right)^{3}\right]$

where $l_{\mathrm{ov}}$ is the length of the overlapping region of the stator and rotor fingers, $\mu_{\mathrm{e}, \mathrm{s}}=\frac{\mu}{1+6 \mathrm{Kn}_{\mathrm{s}}}\left(\mu_{\mathrm{e}, \mathrm{f}}=\right.$ $\left.\frac{\mu}{1+6 \mathrm{Kn}_{\mathrm{f}}}\right)$ is the effective viscosity of the air between the sense (feedback) fingers, $h_{\mathrm{s}}=1.3 g_{\mathrm{s}}\left(1+3.3 \mathrm{Kn}_{\mathrm{s}}\right)\left(h_{\mathrm{f}}=\right.$ $\left.1.3 g_{\mathrm{f}}\left(1+3.3 \mathrm{Kn}_{\mathrm{f}}\right)\right)$ is an empirically determined elongation correction to account for the small aspect ratio $\frac{h}{g_{\mathrm{s}}}\left(\frac{h}{g_{\mathrm{f}}}\right)$ of the air gaps, $\mathrm{Kn}_{\mathrm{s}}=\frac{\lambda}{g_{\mathrm{s}}}\left(\mathrm{Kn}_{\mathrm{f}}=\frac{\lambda}{g_{\mathrm{f}}}\right)$ is the Knudsen number, and $\lambda=6.5 \cdot 10^{-8} \mathrm{~m}$ is the mean free path of air at atmospheric pressure.

\subsubsection{Mechanical spring stiffness}

To compute the mechanical spring coefficients $k_{\mathrm{x}}=\frac{F_{\mathrm{x}}}{u}$, $k_{\mathrm{y}}=\frac{F_{\mathrm{y}}}{v}$, and $k_{\theta}=\frac{M}{\vartheta}$ for inertia forces acting on the center of the proof mass, we use the three-beam model depicted in Fig. 4. For our analysis, we assume that node 3 is fixed (clamped), and a displacement $[u, v, \varphi]$ is forced upon node 4 . Due to symmetry, forces $F_{\mathrm{x}}$ and $F_{\mathrm{y}}$ acting on the proof mass are also acting on node 4 (forces divided by 4 obviously since we have $4 \mathrm{U}$ springs). Therefore, we can find the stiffness in the $x$ and $y$ direction by determining the spring constants of the structure of Fig. 4a by applying different values for $u, v$ and $\varphi$. For the stiffness in $x$ direction, we take $u \neq 0, v=0$, and $\varphi=0$, while for the $y$ direction we use $u=0, v \neq 0$, and $\varphi=0$.

For each U-spring we compute the stiffness matrix

$$
\tilde{\mathbf{K}}_{44}=\left[\begin{array}{ccc}
K_{\mathrm{x}} & K_{\mathrm{xy}} & K_{\mathrm{x} \varphi} \\
K_{\mathrm{xy}} & K_{\mathrm{y}} & K_{\mathrm{y} \varphi} \\
K_{\mathrm{x} \varphi} & K_{\mathrm{y} \varphi} & K_{\varphi}
\end{array}\right]
$$


that relates the displacements $u$ and $v$ and rotation $\phi$ of node 4 to the applied forces $X_{4}, Y_{4}$, and $M_{4}$ at that node. This stiffness matrix can be determined straightforwardly from linear beam theory. The analysis models for determining the stiffness matrix $\tilde{\mathbf{K}}_{44}$ are given in the Appendix. Since we have 4 identical Usprings, the total stiffness constants in $x$ and $y$ directions are given by $k_{\mathrm{x}, \mathrm{m}}=4 K_{\mathrm{x}}$ and $k_{\mathrm{y}}=4 K_{\mathrm{y}}$, where we use the suffix $m$ to differentiate between the mechanical spring stiffness and the electrostatic contribution $k_{\mathrm{x}, \mathrm{e}}$.

The rotational stiffness $k_{\theta}$ requires a different approach since the center of rotation is at the center of the proof mass, and not at node 4 . We use a simple model to translate a rotation $\vartheta$ at the center of the proof mass into displacements and rotation of node 4. Assuming small rotations and a rigid proof mass gives $\mathbf{u}_{4}=[u, v, \varphi]=\left[\frac{w_{\mathrm{p}}}{2} \vartheta, \frac{l_{\mathrm{p}}}{2} \vartheta, \vartheta\right]=$ $\mathbf{p} \vartheta$, where $\mathbf{p}=\left[\frac{w_{\mathrm{p}}}{2}, \frac{l_{\mathrm{p}}}{2}, 1\right]^{T}$ (Fig. 4c). Similarly, the forces $\mathbf{F}_{4}=\left[X_{4}, Y_{4}, M_{4}\right]$ acting on node 4 generate a moment $M=\frac{w_{\mathrm{p}}}{2} X_{4}+\frac{l_{\mathrm{p}}}{2} Y_{4}+M_{4}=\mathbf{p}^{T} \mathbf{F}_{4}$ around the center of the proof mass. From (88) and by neglecting cross-axis sensitivities $\left(K_{\mathrm{xy}}=0\right)$ we have for the reaction forces $\mathbf{F}_{4}=\left[X_{4}, Y_{4}, M_{4}\right]^{T}=\tilde{\mathbf{K}}_{44} \mathbf{u}_{4}=\tilde{\mathbf{K}}_{44} \mathbf{p} \vartheta$. With $M=\mathbf{p}^{T} \mathbf{F}_{4}=\mathbf{p}^{T} \tilde{\mathbf{K}}_{44} \mathbf{p} \vartheta$, the torsional stiffness $K_{\theta}=$ $M / \vartheta$ for a single spring is given by $K_{\theta}=\mathbf{p}^{T} \tilde{\mathbf{K}}_{44} \mathbf{p}$. Since we have 4 identical U-springs, the total rotational stiffness is given by $k_{\theta}=4 K_{\theta}$.

\subsubsection{Design constraints}

The constraints of the Structures discipline are used to have enough room between the various parts of the device, and to prevent unwanted geometries. Constraint $g_{\mathrm{s}, 1}$ limits the length of the device to be below $l_{\max }\left(l_{\mathrm{p}}+2\left(l_{\mathrm{b} 2}+l_{\mathrm{a}}\right) \leq l_{\max }\right):$

$g_{\mathrm{s}, 1}=\frac{l_{\mathrm{p}}+2\left(l_{\mathrm{b} 2}+l_{\mathrm{a}}\right)}{l_{\max }}-1 \leq 0$

The width measured either at the comb or spring is constrained to be below $w_{\max }$ by constraints $g_{\mathrm{s}, 2}\left(w_{\mathrm{p}}+\right.$ $\left.2\left(l_{\mathrm{f}}+l_{\mathrm{s} 2}+w_{\mathrm{sa}}\right) \leq w_{\max }\right)$, and $g_{\mathrm{s}, 3}\left(w_{\mathrm{p}}+2 l_{\mathrm{b} 3} \leq w_{\max }\right)$ :

$g_{\mathrm{s}, 2}=\frac{w_{\mathrm{p}}+2\left(l_{\mathrm{f}}+l_{\mathrm{s} 2}+w_{\mathrm{sa}}\right)}{w_{\max }}-1 \leq 0$

$g_{\mathrm{s}, 3}=\frac{w_{\mathrm{p}}+2 l_{\mathrm{b} 3}}{w_{\max }}-1 \leq 0$

Constraint $g_{\mathrm{s}, 4}$ assures that beam 2 is long enough to incorporate the limit stop plus its required gap $\left(l_{\mathrm{b} 2} \geq\right.$ $\left.g_{\mathrm{x}}+l_{\mathrm{ls}}\right)$ :

$g_{\mathrm{s}, 4}=10^{5}\left(g_{\mathrm{x}}+l_{\mathrm{ls}}-l_{\mathrm{b} 2}\right) \leq 0$
The large factors in the above constraint, and constraints $g_{\mathrm{s}, 5}-g_{\mathrm{s}, 13}, g_{\mathrm{s}, 15}$, and $g_{\mathrm{s}, 18}$, are used for scaling purposes (we scaled in this way to preserve the linearity of the constraints). Constraint $g_{\mathrm{s}, 5}$ requires beam 1 of the U-spring to leave enough room for the spring anchor $\left(w_{\mathrm{p}}+2 l_{\mathrm{b} 3} \geq w_{\mathrm{a}}+2 l_{\mathrm{b} 1}\right)$ :

$g_{\mathrm{s}, 5}=10^{5}\left(w_{\mathrm{a}}+2 l_{\mathrm{b} 1}-w_{\mathrm{p}}-2 l_{\mathrm{b} 3}\right) \leq 0$

Contact between the stator fingers and the proof mass is prevented by constraining the limit stop gap $g_{\mathrm{y}}$ with $g_{\mathrm{s}, 6}\left(g_{\mathrm{y}} \leq 0.9\left(l_{\mathrm{f}}-l_{\mathrm{ov}}\right)\right)$ :

$g_{\mathrm{s}, 6}=10^{6}\left(g_{\mathrm{y}}-0.9\left(l_{\mathrm{f}}-l_{\mathrm{ov}}\right)\right) \leq 0$

Constraint $g_{\mathrm{s}, 7}$ makes sure that the anchor for the short stator fits between the two stator fingers $\left(l_{\mathrm{sa}} \leq w_{\mathrm{s}}+\right.$ $\left.g_{\mathrm{s}}+w_{\mathrm{f}}\right)$ :

$g_{\mathrm{s}, 7}=10^{6}\left(l_{\mathrm{sa}}-w_{\mathrm{s}}-g_{\mathrm{s}}-w_{\mathrm{f}}\right) \leq 0$

The $x$ limit is constrained to be smaller than both $g_{\mathrm{s}}$ and $g_{\mathrm{f}}$ through $g_{\mathrm{s}, 8}\left(g_{\mathrm{x}} \leq 0.9 g_{\mathrm{s}}\right)$, and $g_{\mathrm{s}, 9}\left(g_{\mathrm{x}} \leq 0.9 g_{\mathrm{f}}\right)$ :

$g_{\mathrm{s}, 8}=10^{6}\left(g_{\mathrm{x}}-0.9 g_{\mathrm{s}}\right) \leq 0$

$g_{\mathrm{s}, 9}=10^{6}\left(g_{\mathrm{x}}-0.9 g_{\mathrm{f}}\right) \leq 0$

Constraint $g_{\mathrm{s}, 10}$ makes sure that the limit stops are on the proof mass and not on the spring beams $\left(w_{\mathrm{p}} / 2 \geq\right.$ $\left.w_{\mathrm{sb}} / 2+g_{\mathrm{y}}+w_{\mathrm{ls}}\right)$ :

$g_{\mathrm{s}, 10}=10^{4}\left(w_{\mathrm{sb}} / 2+g_{\mathrm{y}}+w_{\mathrm{ls}}-w_{\mathrm{p}} / 2\right) \leq 0$

Constraints $g_{\mathrm{s}, 11}, g_{\mathrm{s}, 12}$, and $g_{\mathrm{s}, 13}$ restrict the aspect ratios of the U-spring beams to be below 70 to prevent extremely slender beams that are likely to buckle:

$g_{\mathrm{s}, 11}=10^{4}\left(l_{\mathrm{b} 1}-70 w_{\mathrm{b}}\right) \leq 0$

$g_{\mathrm{s}, 12}=10^{4}\left(l_{\mathrm{b} 2}-70 w_{\mathrm{b} 2}\right) \leq 0$

$g_{\mathrm{s}, 13}=10^{4}\left(l_{\mathrm{b} 3}-70 w_{\mathrm{b}}\right) \leq 0$

Spring clearance constraint $g_{\mathrm{s}, 14}$ assures that the gap between the U-spring beams and the first stator finger is at least $g_{\min }$ :

$$
\begin{aligned}
g_{\mathrm{s}, 14}= & -\frac{1}{2 g_{\min }}\left[l_{\mathrm{p}}-2 w_{\mathrm{b}}-2 g_{\mathrm{sf}}\right. \\
& -N_{\mathrm{f}} / 2\left(w_{\mathrm{f}}+2 w_{\mathrm{s}}+2 g_{\mathrm{f}}\right)-2\left(N_{\mathrm{f}} / 2-1\right) g_{\mathrm{fu}} \\
& \left.-N_{\mathrm{s}} / 2\left(w_{\mathrm{f}}+2 w_{\mathrm{s}}+2 g_{\mathrm{s}}\right)-\left(N_{\mathrm{s}} / 2-1\right) g_{\mathrm{su}}\right]+1 \leq 0
\end{aligned}
$$


Constraint $g_{\mathrm{s}, 15}$ assures that the width of the stopping block does not exceed the width of the U-spring anchor:

$g_{\mathrm{s}, 15}=10^{5}\left(w_{\mathrm{sb}}-w_{\mathrm{a}}\right) \leq 0$

Constraints $g_{\mathrm{s}, 16}$ and $g_{\mathrm{s}, 17}$ constrain the area to be at most $A_{\max }$, where the width is measured at the fingers $\left(g_{\mathrm{s}, 16}\right)$ and the anchors $\left(g_{\mathrm{s}, 17}\right)$ :

$g_{\mathrm{s}, 16}=\frac{\left(l_{\mathrm{p}}+2\left(l_{\mathrm{b} 2}+l_{\mathrm{a}}\right)\right)\left(w_{\mathrm{p}}+2\left(l_{\mathrm{f}}+l_{\mathrm{s} 2}+w_{\mathrm{sa}}\right)\right)}{A_{\max }}-1 \leq 0$

$g_{\mathrm{s}, 17}=\frac{\left(l_{\mathrm{p}}+2\left(l_{\mathrm{b} 2}+l_{\mathrm{a}}\right)\right)\left(w_{\mathrm{p}}+2 l_{\mathrm{b} 3}\right)}{A_{\max }}-1 \leq 0$

Constraint $g_{\mathrm{s}, 18}$ limits the length of beam 2 to be at least 3 times the gap $g_{\mathrm{x}}$ in $\mathrm{x}$-direction:

$g_{\mathrm{s}, 18}=10^{6}\left(3 g_{\mathrm{x}}-l_{\mathrm{b} 2}\right) \leq 0$

The initial values of the design parameters are listed in Table 2.

\subsection{Electrostatics}

In subsystem Electrostatics, we use analytical models from Senturia (2001) to determine the spring softening effect, the differential capacitance sensitivity, the parasitic capacitance, and the maximally detectable acceleration as functions of the dimensions of the fingers and gaps, and the modulation voltage.

\subsubsection{Differential capacitance sensitivity and parasitic capacitance}

To determine the at-rest sensing and feedback capacitances $C_{\mathrm{s}}$ and $C_{\mathrm{f}}$, we use parallel-plate estimates (Senturia 2001). A single stator-rotor-stator finger cell as depicted in Fig. 5 can be seen as two parallel plate capacitors, where $V_{\mathrm{s}}$ is the modulation voltage. With
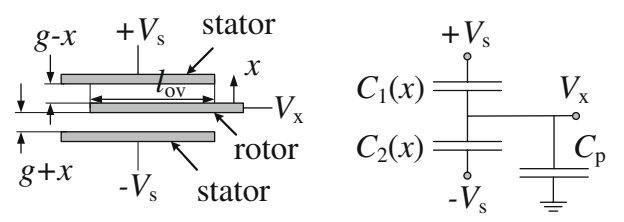

Fig. 5 Single stator-rotor-stator cell (left) and equivalent circuit (right) all capacitors connected in parallel, the capacitance for the $N_{\mathrm{s}}$ sense cells as a function of the displacement $x$ is

$C_{\mathrm{s}, 1}(x)=N_{\mathrm{s}} \frac{\varepsilon l_{\mathrm{ov}} h}{g_{\mathrm{s}}-x} \quad C_{\mathrm{s}, 2}(x)=N_{\mathrm{s}} \frac{\varepsilon l_{\mathrm{ov}} h}{g_{\mathrm{s}}+x}$

where $\varepsilon=8.859 \cdot 10^{-12} \mathrm{~F} / \mathrm{m}$ is the permittivity of air, $l_{\text {ov }}$ the overlap length, $h$ the structural height, and $g_{\mathrm{s}}$ the nominal gap between the stator and rotor fingers. $C_{\mathrm{s}, 1}$ is associated with the gaps that become smaller for increasing $x$, and $C_{\mathrm{s}, 2}$ for the increasing gaps. Note that the contributions of the fringing fields have been neglected here.

The nominal at-rest capacitance $C_{\mathrm{s}}$ for the sense units is given by

$C_{\mathrm{s}}=C_{\mathrm{s}, 1}(x=0)=C_{\mathrm{s}, 2}(x=0)=N_{\mathrm{s}} \frac{\varepsilon l_{\mathrm{ov}} h}{g_{\mathrm{s}}}$

Similarly, the at-rest capacitance for the feedback units is given by

$C_{\mathrm{f}}=N_{\mathrm{f}} \frac{\varepsilon l_{\mathrm{ov}} h}{g_{\mathrm{f}}}$

The differential capacitance sensitivity $S_{\mathrm{d}}=\frac{\Delta C(x)}{x}$ in $[\mathrm{F} / \mathrm{m}]$ is defined as the ratio between the differential capacitance change $\Delta C(x)$ and the displacement $x$ of the proof mass. For small movements $x$, the differential capacitance $\Delta C(x)=C_{\mathrm{s}, 1}(x)-C_{\mathrm{s}, 2}(x)$ is approximated by

$$
\begin{aligned}
\Delta C(x) & =C_{\mathrm{s}, 1}(x)-C_{\mathrm{s}, 2}(x)=C_{\mathrm{s}} \frac{g_{\mathrm{s}}}{g_{\mathrm{s}}-x}-C_{\mathrm{s}} \frac{g_{\mathrm{s}}}{g_{\mathrm{s}}+x} \\
& =\frac{2 C_{\mathrm{s}} g_{\mathrm{s}} x}{g_{\mathrm{s}}^{2}-x^{2}} \approx \frac{2 x C_{\mathrm{s}}}{g_{\mathrm{s}}}
\end{aligned}
$$

and the differential capacitance sensitivity $S_{\mathrm{d}}$ in $[\mathrm{F} / \mathrm{m}]$ becomes

$S_{\mathrm{d}}=\frac{\Delta C(x)}{x}=\frac{2 C_{\mathrm{s}}}{g_{\mathrm{s}}}$

The parasitic capacitance $C_{\mathrm{p}}$ from the proof mass and rotor fingers to the substrate is determined using a parallel plate estimate as well

$C_{\mathrm{p}}=\frac{\varepsilon l_{\mathrm{p}} w_{\mathrm{p}}}{d}+\left(N_{\mathrm{s}}+N_{\mathrm{f}}\right) \frac{\varepsilon l_{\mathrm{f}} w_{\mathrm{f}}}{d}$

where $d$ is the air gap height between the suspended structure and the substrate.

\subsubsection{Spring softening, pull-in, and self-test}

When an AC voltage with amplitude $V_{\mathrm{s} 0}$ and a frequency much larger than the measurement frequency is applied to measure the displacement $x$, electrostatic forces on the movable fingers are introduced. Each gap generates a force proportional to the inverse of the square of the gap size. For $x=0$, these forces are 
equal and opposite and cancel each other out. For nonzero displacements however, a net electrostatic force in the direction of $x$ is generated. Since this force is in opposite direction to the spring restoring force, a spring softening effect occurs. For each gap $g$, the magnitude of the electrostatic force $F$ is (Senturia 2001)

$F=\frac{\varepsilon l_{\mathrm{ov}} h V_{\mathrm{s} 0}^{2}}{4 g^{2}}$

The net electrostatic force $F_{\mathrm{e}}$ acting on the movable fingers due to a displacement of size $x$ can be determined from the difference between the forces generated by the two gaps

$F_{\mathrm{e}}=F_{1}-F_{2}=\frac{\varepsilon l_{\mathrm{ov}} h V_{\mathrm{s} 0}^{2}}{4\left(g_{\mathrm{s}}-x\right)^{2}}-\frac{\varepsilon l_{\mathrm{ov}} h V_{\mathrm{s} 0}^{2}}{4\left(g_{\mathrm{s}}+x\right)^{2}}=\frac{C_{\mathrm{s}} V_{\mathrm{s} 0}^{2} g_{\mathrm{s}}^{2} x}{\left(g_{\mathrm{s}}^{2}-x^{2}\right)^{2}}$

For large displacements, the electrostatic force $F_{\mathrm{e}}$ becomes larger when the displacement $x$ increases and tends to infinity as $x \rightarrow g_{\mathrm{s}}$.

The contribution of the electrostatic force $F_{\mathrm{e}}$ can be seen as an additional stiffness $k_{\mathrm{x}, \mathrm{e}}$. Since the force is in the direction opposite to the spring restoring force, the electrostatic force has a spring-softening effect, and the stiffness $k_{\mathrm{x}, \mathrm{e}}$ is negative. The stiffness $k_{\mathrm{x}, \mathrm{e}}$ is a function of the position $x$ and is given by

$k_{\mathrm{x}, \mathrm{e}}(x)=-\frac{d F_{\mathrm{e}}(x)}{d x}=-\frac{C_{\mathrm{s}} V_{\mathrm{s} 0}^{2} g_{\mathrm{s}}{ }^{2}\left(g_{\mathrm{s}}^{2}+3 x^{2}\right)}{\left(g_{\mathrm{s}}{ }^{2}-x^{2}\right)^{3}}$

For small displacements $x \ll g_{\mathrm{s}}$, the spring-softening effect can be approximated by a constant stiffness $k_{\mathrm{x}, \mathrm{e}}^{0}$ defined as

$k_{\mathrm{x}, \mathrm{e}}^{0}=k_{\mathrm{x}, \mathrm{e}}(x=0)=-\frac{C_{s} V_{\mathrm{s} 0}^{2}}{g_{\mathrm{s}}^{2}}$

When an external acceleration in the $x$ direction is applied, the inertial force $F_{\mathrm{x}}=m a_{\mathrm{x}}$ is balanced by the spring restoring force $F_{\mathrm{m}}=k_{\mathrm{x}, \mathrm{m}} x$ and the electrostatic force $F_{\mathrm{e}}$ :

$m a_{\mathrm{x}}=F_{\mathrm{m}}+F_{\mathrm{e}}=k_{\mathrm{x}, \mathrm{m}} x-\frac{C_{\mathrm{s}} V_{\mathrm{s} 0}^{2} g_{\mathrm{s}}^{2} x}{\left(g_{\mathrm{s}}^{2}-x^{2}\right)^{2}}$

Hence, the maximally detectable acceleration due to pull-in $a_{\text {max,pi }}$ occurs where $F_{\mathrm{m}}+F_{\mathrm{e}}$ is maximal. For any acceleration larger than $a_{\mathrm{max}, \mathrm{pi}}$, the spring restoring force will not be large enough to compensate for the inertial and electrostatic forces. The residual force will pull the proof mass into the limit stops, or worse, create contact between the comb fingers. This phenomenon is known as pull-in, and is undesirable.
The force $F_{\mathrm{x}}=F_{\mathrm{m}}+F_{\mathrm{e}}$ is maximal where its derivative is zero:

$$
\frac{d F_{\mathrm{x}}}{d x}=\frac{d F_{\mathrm{m}}}{d x}+\frac{d F_{\mathrm{e}}}{d x}=k_{\mathrm{x}, \mathrm{m}}-\frac{C_{\mathrm{s}} V_{\mathrm{s} 0}^{2} g_{\mathrm{s}}^{2}\left(g_{\mathrm{s}}^{2}+3 x^{2}\right)}{\left(g_{\mathrm{s}}^{2}-x^{2}\right)^{3}}=0
$$

The solution $x_{\text {pull }}$, obtained with Matlab's symbolic solver (Mathworks 2008), is given by

$x_{\text {pull }}= \begin{cases}g_{\mathrm{s}} \sqrt{1+\frac{c}{k}-\frac{1}{c}} & \text { for } \quad k \leq 1 \\ 0 & \text { otherwise }\end{cases}$

where $k=\frac{k_{\mathrm{x}, \mathrm{m}}}{\mid k_{\mathrm{x}, \mathrm{e}}}, c=\left[2 k^{2}\left(\sqrt{1+\frac{1}{4 k}}-1\right)\right]^{\frac{1}{3}}$. This solution is nonsmooth at $k_{\mathrm{x}, \mathrm{m}}=\left|k_{\mathrm{x}, \mathrm{e}}^{0}\right|$. For values of $k_{\mathrm{x}, \mathrm{m}}$ equal to or smaller than this value, the equilibrium at $x=0$ becomes unstable and even the smallest applied acceleration causes pull-in since the net electrostatic force exceeds the spring restoring force for every $x \neq 0$. The maximally detectable acceleration $a_{\text {max,pi }}$ is given by

$a_{\mathrm{max}, \mathrm{pi}}=\frac{k_{\mathrm{x}, \mathrm{m}} x_{\mathrm{pull}}}{m}-\frac{C_{s} V_{\mathrm{s} 0}^{2} g_{\mathrm{s}}^{2} x_{\mathrm{pull}}}{m\left(g_{\mathrm{s}}^{2}-x_{\mathrm{pull}}^{2}\right)^{2}}$

To prevent contact of the comb fingers, limit stops are positioned at both ends of the proof mass. If the gap size $g_{x}$ is smaller than the pull-in displacement $x_{\text {pull }}$, then the maximally detectable acceleration is limited by the maximal deflection $g_{\mathrm{x}}$. The maximally detectable acceleration due to the limit stops is then given by

$a_{\mathrm{max}, \mathrm{ls}}=\frac{k_{\mathrm{x}, \mathrm{m}} g_{\mathrm{x}}}{m}-\frac{C_{\mathrm{s}} V_{\mathrm{s} 0}^{2} g_{\mathrm{s}}^{2} g_{\mathrm{x}}}{m\left(g_{\mathrm{s}}^{2}-g_{\mathrm{x}}^{2}\right)^{2}}$

The feedback fingers are used to apply an electrostatic force $F_{\mathrm{d}}$ on the proof mass to mimic an external acceleration which can be used for self-test. To obtain a linear relation between the applied drive voltage $V_{\mathrm{d}}$ and the generated electrostatic force $F_{\mathrm{d}}$, voltages $V_{\mathrm{c}, \mathrm{d}}+V_{\mathrm{d}}$ and $V_{\mathrm{c}, \mathrm{d}}-V_{\mathrm{d}}$ are applied separately across the two comb finger gaps (Boser and Howe 1995). Here $V_{\mathrm{c}, \mathrm{d}}$ is a fixed center voltage, and $V_{\mathrm{d}}$ is the controlling drive voltage. In this case, the force $F_{\mathrm{d}}$ is given by

$F_{\mathrm{d}}=\frac{2 V_{\mathrm{c}, \mathrm{d}} V_{\mathrm{d}} C_{\mathrm{f}}}{g_{\mathrm{f}}}$

and the maximally acceleration for self-test $a_{\max , \text { st }}$ becomes

$a_{\mathrm{max}, \mathrm{st}}=\frac{F_{\mathrm{d}}}{m}=\frac{2 V_{\mathrm{c}, \mathrm{d}} V_{\mathrm{d}} C_{\mathrm{f}}}{m g_{\mathrm{f}}}$ 


\subsubsection{Design constraints}

The design constraints for subsystem Electrostatics are concerned with avoiding pull-in of the capacitors and requiring a minimal self-test force. Constraint $g_{\mathrm{e}, 1}$ assures that the spring-softening effect does not reduce the stiffness to a point where pull-in occurs already at very low accelerations by constraining the at-rest negative stiffness to be at most $90 \%$ of the mechanical stiffness $\left(-k_{\mathrm{x}, \mathrm{e}} \leq 0.9 k_{\mathrm{x}, \mathrm{m}}\right)$ :

$g_{\mathrm{e}, 1}=-k_{\mathrm{x}, \mathrm{e}}-0.9 k_{\mathrm{x}, \mathrm{m}} \leq 0$

Constraints $g_{\mathrm{e}, 2}$ and $g_{\mathrm{e}, 3}$ make sure that the limit stop is hit before pull-in occurs for large input accelerations $\left(g_{\mathrm{x}} \leq 0.9 x_{\text {pull }}\right)$, and that the sense gap is larger than the pull-in displacement $\left(x_{\text {pull }} \leq 0.9 g_{\mathrm{s}}\right)$ :

$g_{\mathrm{e}, 2}=10^{6}\left(g_{\mathrm{x}}-0.9 x_{\text {pull }}\right) \leq 0$

$g_{\mathrm{e}, 3}=10^{6}\left(x_{\text {pull }}-0.9 g_{\mathrm{s}}\right) \leq 0$

The maximally detectable acceleration $a_{\max }$ is constrained to be below the pull-in acceleration by $g_{\mathrm{e}, 4}\left(a_{\max } \leq a_{\max , \mathrm{pi}}\right)$, and below the limit stop acceleration by $g_{\mathrm{e}, 5}\left(a_{\max } \leq a_{\max , \mathrm{ls}}\right)$ :

$g_{\mathrm{e}, 4}=\left(1-\frac{a_{\text {max }, \mathrm{pi}}}{a_{\max }}\right)\left[\left(\frac{g_{\mathrm{s}}}{10^{-6}}\right)^{2}-\left(\frac{x_{\text {pull }}}{10^{-6}}\right)^{2}\right]^{2} \leq 0$

$g_{\mathrm{e}, 5}=\left(1-\frac{a_{\max , \mathrm{ls}}}{a_{\max }}\right)\left[\left(\frac{g_{\mathrm{s}}}{10^{-6}}\right)^{2}-\left(\frac{g_{\mathrm{x}}}{10^{-6}}\right)^{2}\right]^{2} \leq 0$

Constraint $g_{\mathrm{e}, 6}$ requires that the maximally self-testable acceleration is at least equal to the desired testable acceleration $a_{\mathrm{st}}\left(a_{\mathrm{max}, \mathrm{st}} \geq a_{\mathrm{st}}\right)$ :

$g_{\mathrm{e}, 6}=-\frac{a_{\mathrm{max}, \mathrm{st}}}{a_{\mathrm{st}}}+1 \leq 0$

Constraints $g_{\mathrm{e}, 2}$ and $g_{\mathrm{e}, 3}$ together with the last terms in constraints $g_{\mathrm{e}, 4}$ and $g_{\mathrm{e}, 5}$ are used to avoid the singularity in $a_{\text {max,pi }}$ and $a_{\text {max,ls }}$ that arises for $g_{\mathrm{s}}=x_{\text {pull }}$ and $g_{\mathrm{s}}=$ $g_{\mathrm{x}}$, respectively. Note that constraint $g_{\mathrm{e}, 4}$ will never be active since the limit stop will be hit before pull-in can occur. The constraint is only included here for completeness. The initial values of the design parameters are listed in Table 2.

\subsection{Dynamics}

In subsystem Dynamics, a lumped parameter model taken from Mukherjee et al. (1999) is used to evaluate the dynamical characteristics of the accelerometer. The accelerometer is modeled as a mass-spring-dashpot system as illustrated in Fig. 6 from which resonance frequencies, mechanical sensitivity, and mechanical noise can be determined.

\subsubsection{Resonance frequencies, bandwidth, quasi-static sensitivity, and noise}

The model parameters are the effective mass $m$ and the rotational inertia $J$ of the proof mass and rotor fingers, the spring constants $k_{\mathrm{x}}$ and $k_{\mathrm{y}}$ of the $\mathrm{U}$-springs in $x$ and $y$ directions respectively, and the rotational stiffness of the U-springs $k_{\theta}$ around the center of the proof mass. Note that the stiffness in the $x$ direction includes the electrostatic spring softening effect $k_{\mathrm{x}}=k_{x, \mathrm{~m}}+k_{x, \mathrm{e}}^{0}$.

The resonance frequencies in $[\mathrm{rad} / \mathrm{s}]$ in $x, y$, and $\vartheta$ directions are given by

$\omega_{\mathrm{x}}=\sqrt{\frac{k_{\mathrm{x}}}{m}} \quad \omega_{\mathrm{y}}=\sqrt{\frac{k_{\mathrm{y}}}{m}} \quad \omega_{\theta}=\sqrt{\frac{k_{\theta}}{J}}$

The so-called quality factor $Q$ related to the damping of the movement in the $x$ direction (dimensionless) is

$Q=\frac{\omega_{\mathrm{x}} m}{b}$

The device is designed to operate under quasi-static conditions such that the proof mass displacement follows the time-evolution of the applied acceleration. Therefore, one designs the accelerometer to have a resonant frequency much larger than the expected maximum frequency component of the acceleration signal (Senturia 2001). Under quasi-static conditions, the time derivatives of $x$ are zero for frequencies well below resonance $\left(\omega \ll \omega_{\mathrm{x}}\right)$, and the mechanical sensitivity $S_{\mathrm{m}}$ in $\left[\mathrm{m} /\left(\mathrm{m} / \mathrm{s}^{2}\right)\right]$ of the displacement $x$ resulting from an applied acceleration $a_{\mathrm{x}}$ is given by

$S_{\mathrm{m}}=\frac{x}{a_{\mathrm{x}}}=\frac{m}{k_{\mathrm{x}}}=\frac{1}{\omega_{\mathrm{x}}^{2}}$

Note that the sensitivity $S_{\mathrm{m}}$ is inversely proportional to the square of the resonance frequency. For accelerometers that respond quickly, and hence have a high resonance frequency, the amplitude of the position signal to be sensed will be small.
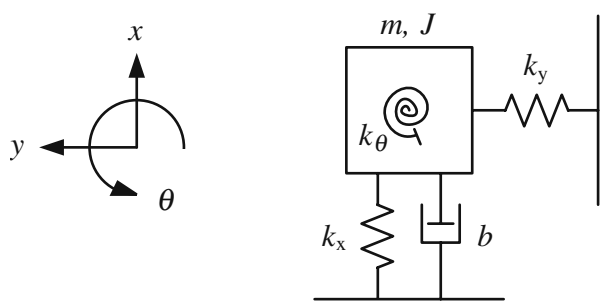

Fig. 6 Mass-spring-dashpot model for the accelerometer 
The $-3 \mathrm{~dB}$ (or half-power) cut-off frequency $\omega_{-3 \mathrm{~dB}}$ defining the mechanical bandwidth of the system is given by

$\omega_{-3 \mathrm{~dB}}=\omega_{\mathrm{x}} \sqrt{1-\frac{1}{2 Q^{2}}+\frac{1}{2 Q^{2}} \sqrt{1-4 Q^{2}+8 Q^{4}}}$

To convert all angular frequencies $\omega$ in $[\mathrm{rad} / \mathrm{s}]$ to frequencies $f$ in $[\mathrm{Hz}]$, divide by $2 \pi$.

In the mechanical domain, random collision of air molecules with the accelerometer introduces so called Brownian (thermal) noise. This noise can be transformed into an equivalent acceleration $a_{\mathrm{n}, \mathrm{m}}$ of (Senturia 2001)

$a_{\mathrm{n}, \mathrm{m}}=\sqrt{\frac{4 k_{\mathrm{B}} T b}{m}} \sqrt{\frac{\omega}{2 \pi}}$

where $k_{\mathrm{B}}=1.28 \cdot 10^{-23} \mathrm{~m}^{2} \mathrm{kgs}^{-2} \mathrm{~K}^{-1}$ is the Boltzmann constant, $T=298 \mathrm{~K}$ is the absolute temperature, and $\omega$ in $[\mathrm{rad} / \mathrm{s}]$ is the frequency range over which the noise contributes. Together with the electronic noise (see next section), the mechanical noise limits the minimally detectable acceleration.

\subsubsection{Design constraints}

The constraints of subsystem Dynamics are mainly used to guarantee appropriate operating conditions for the sense mode. In-plane mode decoupling constraints $g_{\mathrm{d}, 1}$ and $g_{\mathrm{d}, 2}$ assure that the resonant mode in $x$ dominates the modes in $y\left(\omega_{\mathrm{x}} \leq 0.1 \omega_{\mathrm{y}}\right)$, and $\theta\left(\omega_{\mathrm{x}} \leq 0.1 \omega_{\theta}\right)$ :

$g_{\mathrm{d}, 1}=10^{-5}\left(\omega_{\mathrm{x}}-0.1 \omega_{\mathrm{y}}\right) \leq 0$

$g_{\mathrm{d}, 2}=10^{-5}\left(\omega_{\mathrm{x}}-0.1 \omega_{\theta}\right) \leq 0$

where the factors in front of the constraints are used for scaling. Constraint $g_{\mathrm{d}, 3}$ requires the mechanical bandwidth $\omega_{-3 \mathrm{~dB}}$ to be at least a minimal value $\omega_{\min }$ $\left(\omega_{-3 \mathrm{~dB}} \geq \omega_{\min }\right)$ :

$g_{\mathrm{d}, 3}=-\frac{\omega_{-3 \mathrm{~dB}}}{\omega_{\min }}+1 \leq 0$

Quasi-static operating conditions are enforced by constraint $g_{\mathrm{d}, 4}$, which sets the measuring frequency $\omega$ to be well below the resonant frequency $\omega_{\mathrm{x}}\left(\omega \leq 0.1 \omega_{\mathrm{x}}\right)$ :

$g_{\mathrm{d}, 4}=-\frac{0.1 \omega_{\mathrm{x}}}{\omega}+1 \leq 0$

The initial values of the design parameters are again listed in Table 2.

\subsection{Circuit}

In subsystem Circuit, we consider the conditioning circuit that is used for transforming the differential capacitance into an output voltage. The conditioning circuit, which is partly taken from Senturia (2001), consists of a voltage source, the differential capacitor sensing element, a charge-amplifier, an additional non-inverting amplifier, a synchronous demodulator, and a low-pass filter. The variables relevant for the electronics models include resistance, capacitance, and voltage values. From these values, we can determine the sensitivity of the output with respect to a change in the differential capacitance, and quantify measurement noise.

\subsubsection{Circuit components}

The layout of the conditioning circuit is depicted in Fig. 7. The circuit consists of an AC voltage source $V_{\mathrm{s}}$ with amplitude $V_{\mathrm{s} 0}$ in [V] and frequency $\omega_{\mathrm{s}}$ in $[\mathrm{rad} / \mathrm{s}]$. The generated signal as a function of the time $t$ in seconds is given by

$V_{\mathrm{s}}(t)=V_{\mathrm{s} 0} \cos \omega_{\mathrm{s}} t$

To amplify the differential capacitance $\Delta C(x)$ of the sensing element, we use a charge amplifier with feedback capacitance $C_{\mathrm{a}}$. The charge amplifier output voltage $V_{\mathrm{ca}}$ is then (Senturia 2001)

$V_{\text {ca }}(t, x)=\frac{\Delta C(x)}{C_{\mathrm{a}}} V_{\mathrm{s}}(t)$

We assume that the feedback resistance $R_{\mathrm{a}}$ is chosen large enough to make $\omega R_{\mathrm{a}} C_{\mathrm{a}}$ large compared to unity.

In the second stage of amplification, we use a noninverting amplifier with a gain $G_{\mathrm{ni}}$ and ground resistor $R_{\mathrm{ni}}$. The output voltage $V_{\mathrm{a}}$ after the second stage of amplification is then

$V_{\mathrm{a}}(t, x)=G_{\mathrm{ni}} V_{\mathrm{ca}}(t, x)=G_{\mathrm{ni}} \frac{\Delta C(x)}{C_{\mathrm{a}}} V_{\mathrm{s}}(t)$

As can be observed from (65), the amplitude of the high-frequency $V_{\mathrm{a}}(t, x)$ signal is proportional to the differential capacitance $\Delta C(x)$. To extract this amplitude in a way that can follow the relatively slow variations

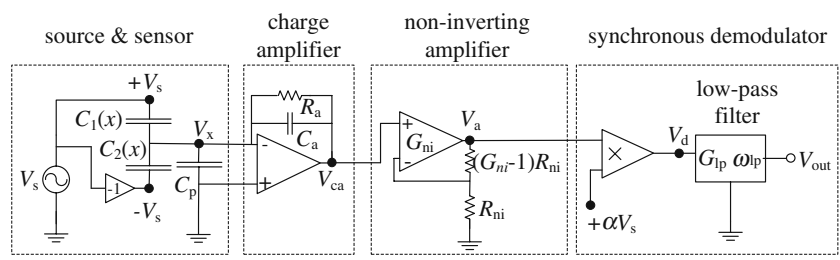

Fig. 7 Schematic of the conditioning circuit 
associated with the changing of $\Delta C(x)$, we use a synchronous demodulator. The demodulator consists of an analog multiplier of two signals: the amplified measurement signal $V_{\mathrm{a}}(t, x)$, and a reference signal $\alpha V_{\mathrm{s} 0} \cos \omega_{\mathrm{s}} t$, where $\alpha$ can be used for scaling. When the reference signal is in phase with the measurement signal, the result is an output voltage $V_{\mathrm{lp}}(t, x)$ given by

$$
\begin{aligned}
V_{\mathrm{lp}}(t, x) & =V_{\mathrm{a}}(t, x) \times \alpha V_{\mathrm{s}}(t) \\
& =\left[G_{\mathrm{ni}} \frac{\Delta C(x)}{C_{\mathrm{a}}} V_{\mathrm{s} 0} \cos \omega_{s} t\right]\left[\alpha V_{\mathrm{s} 0} \cos \omega_{\mathrm{s}} t\right] \\
& =\alpha G_{\mathrm{ni}} \frac{\Delta C(x)}{C_{\mathrm{a}}} V_{\mathrm{s} 0}^{2}\left[1+\cos 2 \omega_{\mathrm{s}} t\right]
\end{aligned}
$$

If the low-pass filter has a cut-off frequency $\omega \ll \omega_{\text {lp }} \ll$ $2 \omega_{\mathrm{s}}$, the component at $2 \omega_{\mathrm{s}}$ is rejected and the positiondependent component below the bandwidth frequency $\omega$ is not. With a filter gain of $G_{\mathrm{lp}}$, the measured output becomes

$V_{\text {out }}(x)=\alpha G_{\mathrm{ni}} G_{\mathrm{lp}} \frac{\Delta C(x)}{C_{\mathrm{a}}} V_{\mathrm{s} 0}^{2}$

which follows $\Delta C(x)$ naturally.

\subsubsection{Sensitivity, range, and noise}

The sensitivity of the conditioning circuit $S_{\mathrm{c}}$ in $[\mathrm{V} / \mathrm{F}]$ follows from

$S_{\mathrm{c}}=\frac{V_{\mathrm{out}}(x)}{\Delta C(x)}=\alpha \frac{G_{\mathrm{ni}} G_{\mathrm{lp}}}{C_{\mathrm{a}}} V_{\mathrm{s} 0}^{2}$

With the mechanical sensitivity $S_{\mathrm{m}}$ and the differential capacitance sensitivity $S_{\mathrm{d}}$, the total measurement sensitivity $S$ in $\left[\mathrm{V} /\left(\mathrm{m} / \mathrm{s}^{2}\right)\right]$ of $V_{\text {out }}$ with respect to an applied acceleration $a$ becomes

$S=\frac{V_{\text {out }}}{a}=\frac{V_{\text {out }}}{\Delta C} \frac{\Delta C}{x} \frac{x}{a}=S_{\mathrm{c}} S_{\mathrm{d}} S_{\mathrm{m}}$

where $x$ in $[\mathrm{m}]$ is the deflection of the proof mass due to an applied acceleration $a$.

The maximally measurable acceleration (the fullscale range $a_{\mathrm{fs}}$ in $\left[\mathrm{m} / \mathrm{s}^{2}\right]$ ) for a sweep from $-0.8 V_{\mathrm{s} 0}$ to $+0.8 V_{\mathrm{s} 0}$ depends on the supply voltage $V_{\mathrm{s} 0}$ and the total sensitivity $S$, and is given by:

$a_{\mathrm{fs}}=\frac{0.8 V_{\mathrm{s} 0}}{S}$

The factor 0.8 keeps the signal away from the saturation levels of the amplifiers.

In the conditioning circuit, an output noise voltage with root-mean-square (RMS) average $V_{\text {n,th }}$ is caused by thermal noise in the resistors. Together with the RMS Brownian noise $a_{\mathrm{n}, \mathrm{m}}$ computed at the structures subsystem, an equivalent acceleration due to noise $a_{\mathrm{n}}$ in $\left[\mathrm{m} / \mathrm{s}^{2}\right]$ is given by (Mukherjee et al. 1999)

$a_{\mathrm{n}}=\sqrt{a_{\mathrm{n}, \mathrm{m}}^{2}+\left(\frac{V_{\mathrm{n}, \mathrm{th}}}{S}\right)^{2} \frac{\omega}{2 \pi}}$

where $\omega$ is the measurement bandwidth. Acceleration values smaller than $a_{\mathrm{n}}$ cannot be distinguished from noise, and therefore $a_{\mathrm{n}}$ determines the minimally detectable acceleration.

\subsubsection{Design constraints}

A total of six design constraints are posed at subsystem Circuit to enforce desired performance of the device after optimization. Constraints $g_{\mathrm{c}, 1}$ and $g_{\mathrm{c}, 2}$ are design constraints that make sure that the accelerometer performs to certain specifications. Constraints $g_{\mathrm{c}, 1}$ sets a lower bound $S_{\min }$ for the total sensitivity of the device $S\left(S \geq S_{\min }\right)$ :

$g_{\mathrm{c}, 1}=-\frac{S}{S_{\min }}+1 \leq 0$

Constraint $g_{\mathrm{c}, 2}$ constrains the noise $a_{\mathrm{n}}$ to be at most the desired minimally detectable acceleration $a_{\min }\left(a_{\mathrm{n}} \leq\right.$ $\left.a_{\min }\right)$ :

$g_{\mathrm{c}, 2}=\frac{a_{\mathrm{n}}}{a_{\min }}-1 \leq 0$

Constraints $g_{\mathrm{c}, 3}$ and $g_{\mathrm{c}, 4}$ make sure that the low-pass filter cut-off frequency is at least 10 times larger than the measurement frequency $\left(\omega \leq 0.1 \omega_{\mathrm{lp}}\right)$, but at most a tenth of the high-frequency component of $V_{\mathrm{d}}$ at $2 \omega_{\mathrm{s}}\left(\omega_{\mathrm{lp}} \leq 0.1 \times 2 \omega_{\mathrm{s}}\right)$ :

$g_{\mathrm{c}, 3}=10^{-3}\left(\omega-0.1 \omega_{\mathrm{lp}}\right) \leq 0$

$g_{\mathrm{c}, 4}=10^{-4}\left(\omega_{\mathrm{lp}}-0.2 \omega_{\mathrm{s}}\right) \leq 0$

The full-scale range of the accelerometer should be at least $a_{\mathrm{fs}} \geq a_{\text {meas }}$, which is assured by constraint $g_{\mathrm{c}, 5}$ :

$g_{\mathrm{c}, 5}=-\frac{a_{\mathrm{fs}}}{a_{\text {meas }}}+1 \leq 0$

Constraint $g_{\mathrm{c}, 6}$ makes sure that the feedback capacitance $C_{\mathrm{a}}$ is at least as large as the parasitic capacitance $C_{\mathrm{p}}$ :

$g_{\mathrm{c}, 6}=10^{13}\left(C_{\mathrm{p}}-C_{\mathrm{a}}\right) \leq 0$

The factors in front of constraints $g_{\mathrm{c}, 3}, g_{\mathrm{c}, 4}$, and $g_{\mathrm{c}, 6}$ are used for proper scaling. 


\subsection{Influence of scaling}

Since the magnitude of design parameters in SI-units can range from $10^{-13}$ for capacitances to $10^{5}$ for resonance frequencies, proper scaling of the problem is vital for numerical optimizers to find accurate solutions. This scaling concerns not only the design variables and constraints, but also the analysis models. The stiffness matrix for example is ill-conditioned when expressed in SI-units since displacements are typically in $\mu \mathrm{m}$ while rotations are of much larger order. The inversion of an ill-conditioned stiffness matrix introduces undesired numerical noise in the analysis results. The way of scaling of the stiffness matrix as described in the Appendix guarantees that the stiffness matrix is well conditioned, and thereby avoids numerical noise.

\section{Design optimization}

In this section, we use the analysis models of the previous sections to formulate a number of design optimization problems that aim at finding optimal settings for input parameters such that desired device behavior is obtained. The formulated problems differ in which parameters are selected as optimization variables. We refer to these as Case 1 through Case 4, respectively. The first case only includes the proof mass and U-spring dimensions as optimization variables, and the largest problem includes 22 design variables. Even though the presented problems are fully analytical, they are nonlinear and non-convex, common difficulties in MDO.

Beside an all-in-one formulation, we also present four problem decompositions. These decompositions apply to each of the four problem cases as mentioned above. The different decompositions illustrate how distributed optimization approaches can be applied to microsystems design problems. As a reference, we solve the problem decompositions with several augmented Lagrangian coordination methods, and compare the results with those obtained with the all-in-one formulation.

\subsection{Baseline design}

The analysis models require that values are assigned to all input parameters. To this end, we define a baseline design inspired by the original ADXL150 accelerometer which is designed for a maximum measurement frequency of $\omega=1000 \mathrm{~Hz}$. Figure 8a depicts the dimensions of the sensing part of the accelerometer. The baseline values for all parameters are given in Table 2. Most dimensions are derived from images of actual devices and additional information from Senturia (2001). Several circuit parameter values are taken from Samuels (1996) and Analog Devices Inc. (1998). The circuit parameter values that could not be retrieved directly $\left(C_{\mathrm{a}}, \alpha, G_{\mathrm{lp}}, \omega_{\mathrm{lp}}, V_{\mathrm{n}, \mathrm{th}}, V_{\mathrm{d}}, V_{\mathrm{c}, \mathrm{d}}\right)$ are chosen such that the performance characteristics of the baseline design are similar to those specified in Samuels (1996); Analog Devices Inc. (1998); Senturia (2001) for the ADXL150. The main performance characteristics of the baseline design are determined from the analysis models and compared to specifications of the ADXL150 in Table 1.

\subsection{All-in-one problem formulation}

The formulation of the optimal design problem typically depends on the intended use of the accelerometer. We select the footprint area $A$ (to which fabrication costs are proportional) as the objective of the design problem. The design constraints make sure that performance with respect to sensitivity, noise, and range is at least as good as the baseline design (i.e. $S_{\min }=S\left(\mathbf{z}_{\text {base }}\right)$, $a_{\text {min }}=a_{\mathrm{n}}\left(\mathbf{z}_{\text {base }}\right)$, and $a_{\text {meas }}=a_{\mathrm{fs}}\left(\mathbf{z}_{\text {base }}\right)$, where $\mathbf{z}_{\text {base }}$ is the baseline design). Formulations for maximizing sensitivity, range, or bandwidth can be set up in a similar fashion. The area-minimization problem is given by

$$
\begin{aligned}
\min _{\mathbf{z}, A_{\max }} & A_{\max } \\
\text { subject to } & g_{\mathrm{c}, 1}=-\frac{S(\mathbf{z})}{S_{\min }}+1 \leq 0 \\
& g_{\mathrm{c}, 2}=\frac{\left.a_{\mathrm{n}} \mathbf{z}\right)}{a_{\min }}-1 \leq 0 \\
& g_{\mathrm{c}, 5}=-\frac{a_{\mathrm{fs}}(\mathbf{z})}{a_{\mathrm{m}}}+1 \leq 0 \\
& \mathbf{g}_{\mathrm{f}}(\mathbf{z}) \leq 0 \\
& \mathbf{z}_{\mathrm{lb}} \leq \mathbf{z} \leq \mathbf{z}_{\mathrm{ub}}
\end{aligned}
$$

where $\mathbf{z}$ are the input parameters that are selected as optimization variables. Parameter $A_{\max }$ of constraints $g_{\mathrm{s}, 16}$ and $g_{\mathrm{s}, 17}$ is included as an artificial optimization variable to avoid the nonsmoothness in the definition of the area $A$ in (1). The functional constraints $\mathbf{g}_{\mathrm{f}}$ assure proper functioning of the device, and include all remaining constraints $\mathbf{g}_{\mathrm{f}}=\left[g_{\mathrm{s}, 1}, \ldots, g_{\mathrm{s}, 17}, g_{\mathrm{e}, 1}, \ldots, g_{\mathrm{e}, 6}\right.$, $\left.g_{\mathrm{d}, 1}, \ldots, g_{\mathrm{d}, 4}, g_{\mathrm{c}, 3}, g_{\mathrm{c}, 4}, g_{\mathrm{c}, 6}\right]$.

Table 1 Main performance characteristics of the baseline design as determined from the analysis models, and the specifications for the ADXL150 (Samuels 1996; Senturia 2001)

\begin{tabular}{llll}
\hline & & Analysis models & Specifications \\
\hline Sensitivity & $S$ & $39 \mathrm{mV} / g$ & $38 \mathrm{mV} / g$ \\
Noise & $a_{\mathrm{n}}$ & $30 \mathrm{mg}$ & $32 \mathrm{mg}$ \\
Range & $a_{\mathrm{fs}}$ & $51 \mathrm{~g}$ & $50 \mathrm{~g}$ \\
Device area & $A$ & $0.20 \mathrm{~mm}^{2}$ & $0.20 \mathrm{~mm}^{2}$ \\
Resonance freq. & $\omega_{\mathrm{x}}$ & $24.1 \mathrm{kHz}$ & $24.7 \mathrm{kHz}$ \\
Quality factor & $Q$ & 6.4 & 5.0 \\
\hline
\end{tabular}

$g=9.8 \mathrm{~m} / \mathrm{s}^{2}$ 
To keep the performance characteristics at least at the same level as the baseline design, we take $\omega=1000$ $\mathrm{Hz}=2000 \pi \mathrm{rad} / \mathrm{s}, S_{\min }=38 \mathrm{mV} / g, a_{\min }=30 \mathrm{mg}$, and $a_{\text {meas }}=50 \mathrm{~g}$. We use $L_{\max }=w_{\max }=700 \mu \mathrm{m}$ to limit the width and length of the sensor region to be within a square with sides of $700 \mu \mathrm{m}$. We set $g_{\min }=10 \mu \mathrm{m}$ to provide enough space for the U-springs. Quasistatic conditions are ensured by setting $\omega_{-3 \mathrm{~dB}, \min }=10 \omega$. The absolute maximal acceleration is set to $a_{\max }=$ $10 a_{\text {meas }}=500 \mathrm{~g}$ to provide a safety margin for shocks, and the required self-test acceleration is set to $20 \%$ of the full scale range: $a_{\mathrm{st}}=0.2 a_{\text {meas }}=10 \mathrm{~g}$.

We investigate a number of choices for the design variables $\mathbf{z}$. For the first case, we take only the variables of the proof mass and U-springs as design variables (components $\mathrm{A}$ of Table 4 only, seven variables). For the second case, we add the sense and feedback unit variables to $\mathbf{z}$ as well $(\mathrm{A}+\mathrm{B}, 16$ variables), giving the optimization algorithm more freedom to find a design with minimal area. For the third case, we also include circuit parameters as design variables $(A+B+C, 20$ variables). The fourth case includes the number of sense and feedback units as variables, resulting in a mixed integer problem $(\mathrm{A}+\mathrm{B}+\mathrm{C}+\mathrm{D}, 22$ variables $)$. An overview of the variables for each component, and their variable bounds are given in Table 4. In all cases, parameters that are not taken as optimization variables are set to their baseline values as given in Table 2. The length of beam $3 l_{\mathrm{b} 3}$ and the length of the limit stop $l_{\mathrm{ls}}$ are included as "elastic" variables to make sure that the various components remain connected. These variables are not included as optimization variables, but their values change with the values of the actual optimization variables $\mathbf{z}$. For instance, the length $l_{\mathrm{b} 3}$ of U-spring beam 3 becomes shorter when the proof mass becomes wider, and its length is therefore given by $l_{\mathrm{b} 3} \equiv l_{\mathrm{b} 1}+$ $w_{\mathrm{a}} / 2-w_{\mathrm{p}} / 2$. Similarly, we have $l_{\mathrm{ls}} \equiv l_{\mathrm{b} 2}-g_{\mathrm{x}}$ for the length of the limit stop.

\subsection{All-in-one results}

Design Cases 1, 2, and 3 are solved with the SQP solver fmincon of Matlab 7.1 with default settings except MaxSQPiter, which is set to 200 to prevent loops that do not terminate in fmincon. For each case, 100 different starting points are selected randomly between $-100 \%$ and $+100 \%$ of the baseline values to determine whether the design problems are multimodal or not, and what their local solutions are. For numerical robustness, design variables $\mathbf{z}$ are scaled with respect to their baseline values $\mathbf{z}_{\text {base }}$ such that $\mathbf{z}_{\text {scaled }}=\mathbf{z} / \mathbf{z}_{\text {base }}$ where the division is taken component-wise.
Table 2 Baseline values for required input parameters

\begin{tabular}{|c|c|c|c|}
\hline \multicolumn{4}{|c|}{ Structures } \\
\hline$h$ & $2.0 \mu \mathrm{m}$ & $g_{\mathrm{s}}$ & $1.3 \mu \mathrm{m}$ \\
\hline$l_{\mathrm{p}}$ & $500 \mu \mathrm{m}$ & $g_{\mathrm{su}}$ & $1.3 \mu \mathrm{m}$ \\
\hline$w_{\mathrm{p}}$ & $50 \mu \mathrm{m}$ & $N_{\mathrm{f}}$ & 12 \\
\hline$l_{\mathrm{b} 1}$ & $125 \mu \mathrm{m}$ & $g_{\mathrm{f}}$ & $1.3 \mu \mathrm{m}$ \\
\hline$l_{\mathrm{b} 2}$ & $6.0 \mu \mathrm{m}$ & $g_{\text {fu }}$ & $1.3 \mu \mathrm{m}$ \\
\hline$l_{\mathrm{b} 3}$ & $105 \mu \mathrm{m}$ & $g_{\text {sf }}$ & $13 \mu \mathrm{m}$ \\
\hline$w_{\mathrm{b}}$ & $2.4 \mu \mathrm{m}$ & $d$ & $1.3 \mu \mathrm{m}$ \\
\hline$w_{\mathrm{b} 2}$ & $4.0 \mu \mathrm{m}$ & $\Delta l_{\mathrm{s} 1}$ & $15 \mu \mathrm{m}$ \\
\hline$g_{\mathrm{x}}$ & $0.7 \mu \mathrm{m}$ & $\Delta l_{\mathrm{s} 2}$ & $25 \mu \mathrm{m}$ \\
\hline$g_{\mathrm{y}}$ & $1.0 \mu \mathrm{m}$ & $w_{\mathrm{s}}$ & $4.0 \mu \mathrm{m}$ \\
\hline$l_{\mathrm{ov}}$ & $114 \mu \mathrm{m}$ & $l_{\mathrm{sa}}$ & $8.0 \mu \mathrm{m}$ \\
\hline$l_{\mathrm{f}}$ & $120 \mu \mathrm{m}$ & $w_{\mathrm{sa}}$ & $15 \mu \mathrm{m}$ \\
\hline$w_{\mathrm{f}}$ & $4.0 \mu \mathrm{m}$ & $l_{\mathrm{a}}$ & $7.0 \mu \mathrm{m}$ \\
\hline$N_{\mathrm{s}}$ & 42 & $w_{\mathrm{a}}$ & $10 \mu \mathrm{m}$ \\
\hline$l_{\mathrm{ls}}$ & $2.0 \mu \mathrm{m}$ & $w_{\mathrm{sb}}$ & $10 \mu \mathrm{m}$ \\
\hline$w_{\mathrm{ls}}$ & $2.0 \mu \mathrm{m}$ & & \\
\hline \multicolumn{2}{|c|}{ Electrostatics } & \multicolumn{2}{|c|}{ Dynamics } \\
\hline$\overline{V_{\mathrm{s} 0}}$ & $2.5 \mathrm{~V}$ & $J$ & $5.310^{-6} \mathrm{~kg}\left(\mu \mathrm{m}^{2}\right)^{*}$ \\
\hline$k_{\mathrm{x}, \mathrm{m}}$ & $5.6 \mathrm{~N} / \mathrm{m}^{*}$ & $b$ & $5.5 \mu \mathrm{Ns} / \mathrm{m}^{*}$ \\
\hline$m$ & $0.23 \mu \mathrm{g}^{*}$ & $k_{\mathrm{x}, \mathrm{e}}$ & $-0.24 \mathrm{~N} / \mathrm{m}^{*}$ \\
\hline$V_{\mathrm{d}}$ & $2.5 \mathrm{~V}$ & $k_{\mathrm{y}}$ & 694 N/m* \\
\hline \multirow[t]{2}{*}{$V_{\mathrm{c}, \mathrm{d}}$} & $2.5 \mathrm{~V}$ & $k_{\theta}$ & $45 \mu \mathrm{Nm}^{*}$ \\
\hline & & $\omega$ & $1000 \mathrm{~Hz}$ \\
\hline \multicolumn{2}{|c|}{ Circuit } & \multicolumn{2}{|c|}{ Responses } \\
\hline$\omega_{\mathrm{s}}$ & $100 \mathrm{kHz}$ & $S$ & $39 \mathrm{mV} / \mathrm{g}^{*}$ \\
\hline$C_{\mathrm{a}}$ & $350 \mathrm{fF}$ & $a_{\mathrm{n}}$ & $0.29 \mathrm{~m} / \mathrm{s}^{2 *}$ \\
\hline$C_{\mathrm{p}}$ & $347 \mathrm{fF}^{*}$ & $a_{\mathrm{fs}}$ & $51 g^{*}$ \\
\hline$G_{\mathrm{ni}}$ & 17 & $A$ & $0.21 \mathrm{~mm}^{2} *$ \\
\hline$\alpha$ & 1.0 & $a_{\max , \mathrm{pi}}$ & $1869 g^{*}$ \\
\hline$\omega_{\mathrm{lp}}$ & $15 \mathrm{kHz}$ & $a_{\max , 1 \mathrm{~s}}$ & $1570 g^{*}$ \\
\hline$G_{\mathrm{lp}}$ & 3 & $a_{\max , \mathrm{st}}$ & $79 g^{*}$ \\
\hline$V_{\mathrm{n}, \mathrm{th}}$ & $21 \mu \mathrm{V} / \sqrt{\mathrm{Hz}}$ & $x_{\text {pull }}$ & $0.94 \mu \mathrm{m} *$ \\
\hline$S_{\mathrm{d}}$ & $100 \mathrm{fF} / \mu \mathrm{m}^{*}$ & $\omega_{\mathrm{X}}$ & $24 \mathrm{kHz}^{*}$ \\
\hline$S_{\mathrm{m}}$ & $0.043 \mathrm{~nm} /\left(\mathrm{m} / \mathrm{s}^{2}\right)^{*}$ & $\omega_{\mathrm{y}}$ & $275 \mathrm{kHz}^{*}$ \\
\hline \multirow[t]{3}{*}{$a_{\mathrm{n}, \mathrm{m}}$} & $0.24 \mathrm{~m} / \mathrm{s}^{2 *}$ & $\omega_{\theta}$ & $463 \mathrm{kHz}^{*}$ \\
\hline & & $\omega_{-3 \mathrm{~dB}}$ & $37 \mathrm{kHz} *$ \\
\hline & & $Q$ & $6.4^{*}$ \\
\hline
\end{tabular}

Values marked with an asterisk (*) are computed using the disciplinary analysis models of Sections 3.1-3.4. $g=9.8 \mathrm{~m} / \mathrm{s}^{2}$

Design Case 4 requires a different approach due to the discrete nature of the design variables $N_{\mathrm{s}}$ and $N_{\mathrm{f}}$. We perform an enumerative search in both $N_{\mathrm{s}}=\{2,4$, $6, \ldots, 98,100\}$ and $N_{\mathrm{f}}=\{4,8,12,16,20\}$. For each combination of $N_{\mathrm{s}}$ and $N_{\mathrm{f}}$, we solve 100 optimization problems in the remaining variables of $\mathbf{z}$, each with a different starting point selected randomly between $-100 \%$ and $+100 \%$ of the baseline values. The design with the smallest $A_{\max }$ then gives the optimal solution.

The optimal designs for the four cases are depicted in Fig. 8. The optimal values for the design variables are listed in Table 4. At all solutions, the KarushKuhn-Tucker conditions are satisfied indicating that 
Fig. 8 Baseline and optimal solutions for the minimal area accelerometer design cases

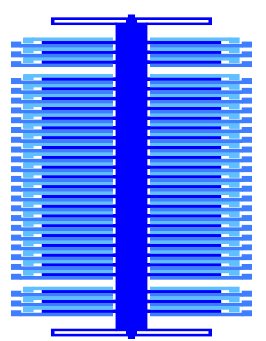

(a) Baseline

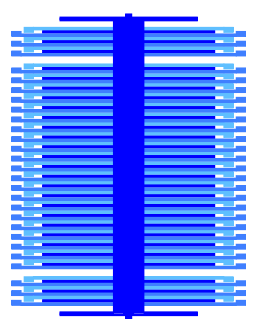

(b) Case 1

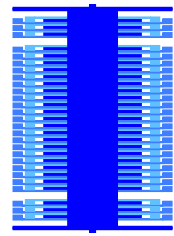

(c) Case 2

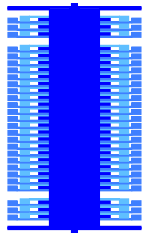

(d) Case 3

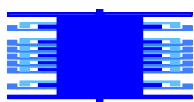

(e) Case 4 the problem is well-defined. As expected, the obtained designs become smaller as the number of design variables increases.

Table 3 summarizes the obtained optimal solutions for each case by listing the percentage of runs that converged, and the smallest, mean, and largest optimal areas for the converged solutions. The active constraints at the optimal design are listed as well. The numbers for Case 4 are based on the 100 starting points with the optimal values for $N_{\mathrm{s}}=10$ and $N_{\mathrm{f}}=4$. The range of the optimal areas is small for all cases, which suggests that the problem may be unimodal, even though the problem functions are non-convex (Table 4).

For Case 1, only a small area reduction is observed. The $6 \%$ decrease in area is gained by shortening the length of beam 2 of the U-spring to its minimally allowed value. The design of Case 2 has considerably shorter comb fingers, and a shorter proof mass. The associated sensitivity loss is compensated by an increase in proof mass width, and a reduction in the sense gap $g_{\mathrm{s}}$. To accommodate all fingers on the shorter proof mass, the spacing gaps $g_{s u}$ and $g_{f u}$ are reduced. Note that the design makes optimal use of the area by making the width at the $\mathrm{U}$-springs equal to the width at the comb fingers. The design of Case 3 has shorter fingers, and the associated loss of sensitivity is compensated by increasing the source voltage and signal amplification in the Circuit subsystem. Finally, Case 4 shows a compact design with fewer sense and feedback fingers, and a shorter proof mass. The loss in sensitivity is compensated by widening the proof mass and elongating the fingers, but also by making the U-springs longer and thus more flexible.

Figure 8 clearly illustrates that the biggest differences are observed for Case 2 and 4 . Adding the dimensions of the comb drive as variables in Case 2 reduces the area with $52 \%$ with respect to the baseline design. Including the circuit parameters as design variables in Case 3 reduces the area by another $8 \%$. When also adding the number of fingers to the optimization variables (Case 4), another $18 \%$ is gained. Overall, the final design for Case 4 is $78 \%$ smaller than the baseline design.

The optimization problems are challenging for fmincon, even though the model functions are all analytical. The SQP solver did not find a feasible solution for many starting points. For all non-successful starting points, fmincon reported "no feasible point found: Magnitude of directional derivative in search direction less than $2 *$ options.TolFun but constraints are not satisfied.". We expect that the linearization of the constraints that fmincon employs has difficulties with the nonlinearity and non-convexity of the analysis models.

\subsection{Four problem decompositions}

Four different problem decompositions are presented to illustrate several ways in which distributed optimization can be used for this design problem. The decompositions also illustrate the various ways in which

Table 3 Number of converged runs, optimal areas in $\mathrm{mm}^{2}$, and active constraints at the optimal design for all-in-one experiments

\begin{tabular}{llllll}
\hline & & Case 1 & Case 2 & Case 3 & Case 4 \\
\hline Converged & & $15 \%$ & $10 \%$ & $15 \%$ & $6 \%$ \\
Area & Min & 0.1878 & 0.0962 & 0.0807 & 0.0451 \\
& Mean & 0.1879 & 0.0963 & 0.0810 & 0.0455 \\
\multicolumn{1}{l}{ Active constraints } & 0.1883 & 0.0964 & 0.0817 & 0.0472 \\
& Max & $g_{s,\{4,5,14,15,17,18\}}$ & $g_{s,\{4,5,6,7,14,15,16,17,18\}}$ & $g_{s,\{4,5,6,7,14,15,16,17,18\}}$ & $g_{s,\{4,5,6,7,11,14,15,16,17,18\}}$ \\
\hline
\end{tabular}


Table 4 Variable selections for the different design cases and optimal values

\begin{tabular}{|c|c|c|c|c|c|c|c|c|c|c|}
\hline \multirow{2}{*}{\multicolumn{2}{|c|}{ Components }} & \multirow{2}{*}{\multicolumn{2}{|c|}{ Variables }} & \multirow{3}{*}{$\begin{array}{l}\text { Lower } \\
0.01\end{array}$} & \multirow{3}{*}{$\begin{array}{r}\text { Upper } \\
1.0\end{array}$} & \multirow{2}{*}{\multicolumn{5}{|c|}{ Optimal designs }} \\
\hline & & & & & & & \multirow{2}{*}{$\begin{array}{r}\text { Case } 1 \\
0.188\end{array}$} & & & \\
\hline $\bar{A}$ & Proof mass \& & $A_{\max }$ & {$\left[\mathrm{mm}^{2}\right]$} & & & Base & & $\begin{array}{r}\text { Case } 2 \\
0.0962\end{array}$ & $\begin{array}{r}\text { Case } 3 \\
0.0807\end{array}$ & $\frac{\text { Case } 4}{0.0449}$ \\
\hline & U-springs & $l_{\mathrm{p}}$ & {$[\mu \mathrm{m}]$} & 2 & 400 & 500 & 477 & 355 & 355 & 133 \\
\hline & & $w_{\mathrm{p}}$ & {$[\mu \mathrm{m}]$} & 2 & 700 & 50 & 49.3 & 80.8 & 81.6 & 139 \\
\hline & & $l_{\mathrm{b} 1}$ & {$[\mu \mathrm{m}]$} & 2 & 400 & 125 & 107 & 124 & 103 & 145 \\
\hline & & $l_{\mathrm{b} 2}$ & {$[\mu \mathrm{m}]$} & 1 & 200 & 6.00 & 2.10 & 1.50 & 1.50 & 1.51 \\
\hline & & $w_{\mathrm{b}}$ & {$[\mu \mathrm{m}]$} & 2 & 10 & 2.40 & 2.00 & 2.00 & 2.00 & 2.07 \\
\hline & & $w_{\mathrm{b} 2}$ & {$[\mu \mathrm{m}]$} & 2 & 10 & 4.00 & 2.03 & 2.00 & 2.00 & 2.00 \\
\hline & & $l_{\mathrm{b} 3} *$ & {$[\mu \mathrm{m}]$} & 2 & 400 & 105 & 87.4 & $88.6^{*}$ & $67.2 *$ & $80.5^{*}$ \\
\hline & & $l_{\mathrm{ls}} *$ & {$[\mu \mathrm{m}]$} & 1 & 100 & 2.00 & 1.40 & $1.00^{*}$ & $1.00 *$ & $1.01^{*}$ \\
\hline \multirow[t]{9}{*}{$\mathrm{B}$} & Comb & $l_{\mathrm{f}}$ & {$[\mu \mathrm{m}]$} & 2 & 400 & 120 & 120 & 38.9 & 17.4 & 30.4 \\
\hline & & $l_{\mathrm{OV}}$ & {$[\mu \mathrm{m}]$} & 2 & 400 & 114 & 114 & 37.8 & 16.3 & 29.2 \\
\hline & & $g_{\mathrm{s}}$ & {$[\mu \mathrm{m}]$} & 0.5 & 20 & 1.30 & 1.30 & 0.84 & 0.80 & 0.80 \\
\hline & & $g_{\text {su }}$ & {$[\mu \mathrm{m}]$} & 0.5 & 20 & 1.30 & 1.30 & 0.50 & 0.50 & 0.50 \\
\hline & & $g_{\mathrm{f}}$ & {$[\mu \mathrm{m}]$} & 0.5 & 20 & 1.30 & 1.30 & 0.79 & 0.95 & 1.89 \\
\hline & & $g_{\mathrm{fu}}$ & {$[\mu \mathrm{m}]$} & 0.5 & 20 & 1.30 & 1.30 & 0.50 & 0.50 & 0.50 \\
\hline & & $g_{\mathrm{X}}$ & {$[\mu \mathrm{m}]$} & 0.5 & 20 & 0.70 & 0.70 & 0.50 & 0.50 & 0.50 \\
\hline & & $w_{\mathrm{f}}$ & {$[\mu \mathrm{m}]$} & 2 & 20 & 4.00 & 4.00 & 5.16 & 5.20 & 5.20 \\
\hline & & $w_{\mathrm{s}}$ & {$[\mu \mathrm{m}]$} & 2 & 20 & 4.00 & 4.00 & 2.00 & 2.00 & 2.00 \\
\hline \multirow[t]{4}{*}{$\mathrm{C}$} & Circuit & $V_{\mathrm{s} 0}$ & {$[\mathrm{~V}]$} & 1 & 4 & 2.50 & 2.50 & 2.50 & 4.00 & 4.00 \\
\hline & & $V_{\mathrm{d}}$ & [V] & 1 & 4 & 2.50 & 2.50 & 2.50 & 2.64 & 3.32 \\
\hline & & $C_{\mathrm{a}}$ & {$[\mathrm{fF}]$} & 1 & 1000 & 350 & 350 & 350 & 231 & 141 \\
\hline & & $G_{\mathrm{ni}}$ & {$[-]$} & 1 & 20 & 17.0 & 17.0 & 17.0 & 20.0 & 20.0 \\
\hline \multirow[t]{2}{*}{$\mathrm{D}$} & Fingers & $N_{\mathrm{s}}$ & {$[-]$} & 2 & 100 & 42 & 42 & 42 & 42 & 10 \\
\hline & & $N_{\mathrm{f}}$ & {$[-]$} & 4 & 20 & 12 & 12 & 12 & 12 & 4 \\
\hline
\end{tabular}

Values in italic are not optimized but inherited from the baseline design. Dependent variables marked with an asterisk $(*)$ are not included in $\mathbf{z}$ but are determined from the definitions $l_{\mathrm{b} 3} \equiv l_{\mathrm{b} 1}+w_{\mathrm{a}} / 2-w_{\mathrm{p}} / 2$ and $l_{\mathrm{ls}} \equiv l_{\mathrm{b} 2}-g_{\mathrm{x}}$

microsystem design problems may be partitioned. Figure 9 depicts the subsystems, and the distribution of variables and functions for each problem decomposition.

The first problem decomposition has three subsystems that each represent a level of abstraction. Figure 9a depicts the distribution of variables and functions over the three subsystems. At the bottom subsystem, the detailed design of the sensing element geometry is taken into account. The middle subsystem considers a lumped parameter model of the sensor dynamics, and the top level includes the sensing element and the electronic circuit. Problem decompositions of this type are commonly referred to as object-based decompositions, and are often hierarchically structured. Note that the variables linking the subsystems are not optimization variables in the all-in-one formulation but intermediate analysis quantities computed by one discipline that are used inputs to another discipline. They appear here as a result of decomposition.

The second decomposition, depicted in Fig. 9b, is a "traditional" multidisciplinary problem decomposition with four subsystems in the sense that it has one subsystem for each analysis discipline. For this decomposition, the linking variables are a mixture of analysis coupling variables, similar to the previous decomposition, and actual design variables of the all-in-one problem.

The third decomposition (Fig. 9c) is object-based and consists of four subsystems. The mid-level Sensor dynamics and top-level Circuit subsystem are identical to their counterparts in the first decomposition. The two lower-level subsystems are associated with the spring design, and the proof mass and fingers designs, respectively. The coupling constraints $g_{\mathrm{s}, 4}, g_{\mathrm{s}, 14}, g_{\mathrm{s}, 16}$, $g_{\mathrm{s}, 17}$, and $g_{\mathrm{s}, 18}$ link the lower-level subsystems.

The fourth problem decomposition has four subsystems with non-separable coupling constraints (Fig. 9d), and is a mixture of an object-based and an aspect-based decomposition. The Circuit subsystem is only coupled to the other three through the coupling constraints, and variable $V_{s 0}$ for Cases 3 and 4 . The variables of subsystem Circuit appear only in Cases 3 and 4. Since its variables do not appear in Cases 1, and 2, the actual optimization of Circuit is omitted for these cases. Note that the analysis models of the Circuit subsystem are still included for the computation of system performance.

In this fourth problem decomposition, subsystems are linked through non-separable coupling constraints. These non-separable functions depend on the design 
Fig. 9 Distribution of variables and function for the four problem decomposition. Single arrows indicate analysis model dependencies, double arrows indicate design variable sharing, and dashed arrows indicate function dependencies

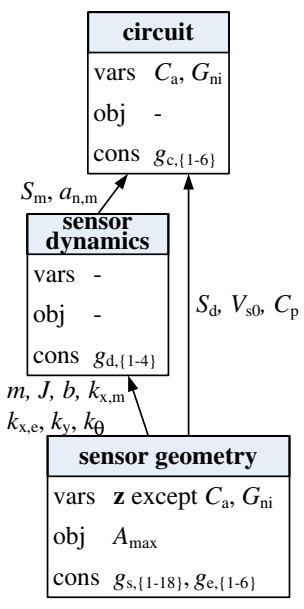

(a) Problem decomposition 1: object-based

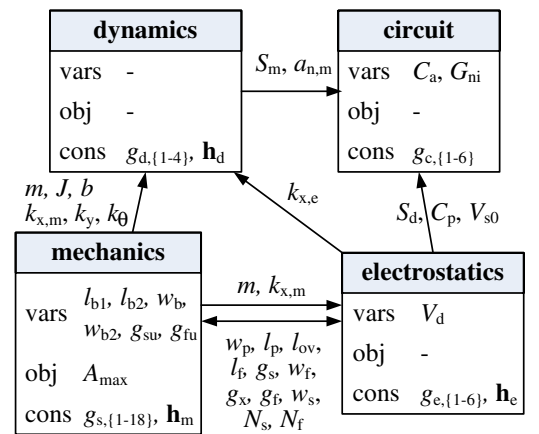

(b) Problem decomposition 2: aspect-based

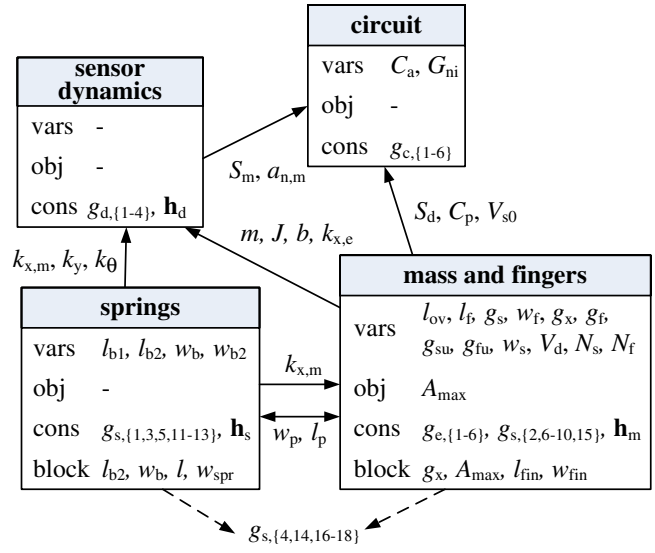

(c) Problem decomposition 3: object-based

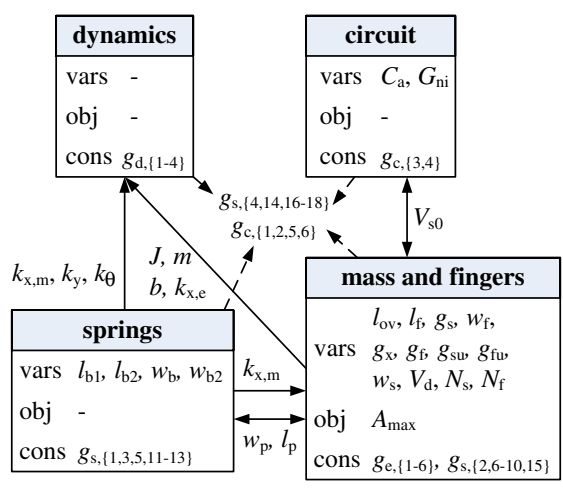

(d) Problem decomposition 4: mixed variables of two or more subsystems. Of these constraints, $g_{\mathrm{s}, 4}, g_{\mathrm{s}, 14}, g_{\mathrm{s}, 16}, g_{\mathrm{s}, 17}, g_{\mathrm{s}, 18}$ depend on variables from the spring and mass subsystems, and constraints $g_{\mathrm{c}, 1}, g_{\mathrm{c}, 2}, g_{\mathrm{c}, 5}$, and $g_{\mathrm{c}, 6}$ link the circuit, dynamics, and mass subsystems.

\subsection{Distributed optimization results using ALC}

This section presents results for augmented Lagrangian coordination approaches (ALC, Tosserams et al. 2006, 2007, 2008, 2009a) that have been used to coordinate the four problem decompositions introduced in the previous section. For each decomposition one appropriate ALC coordination variant is selected. These results may be used as a reference for comparison of coordination algorithms. Note that it is beyond the scope of this paper to describe the ALC method and its implementation for this problem in detail. Our focus is to demonstrate that the benchmark problem has been solved with an actual distributed optimization approach, ALC in this case. For the details of ALC and the four coordination variants, the reader is refer to the references above.

Each problem decomposition of Fig. 9 was solved with an appropriate coordination algorithm from 10 different initial designs selected randomly between $-50 \%$ and $+50 \%$ of the baseline values. For each design, we measured whether the run converged, how many iterations it took (in required number of subproblem optimizations), and what the optimal area of the final design is. ${ }^{1}$

Unless indicated otherwise, termination tolerances for the coordination algorithms were set to $10^{-3}$. For all decompositions we used the initial weight selection strategy as described in Tosserams et al. (2008) with $\alpha=0.1, \mathbf{v}=\mathbf{0}, \mathbf{w}=\mathbf{1 0}^{-\mathbf{3}}$, and $\hat{f}=1$. Furthermore, the

\footnotetext{
${ }^{1} \mathrm{ALC}$ introduces local copies of linking variables at subsystems. Hence the optimal value of a linking variable is taken as the average over these copies. The reader is referred to the aforementioned references for more detail on the use of linking variable copies in ALC.
} 
Table 5 Results for Decomposition 1 using ATC, Decomposition 2 using ALC-QS, and Decomposition 3 using ALC-BS

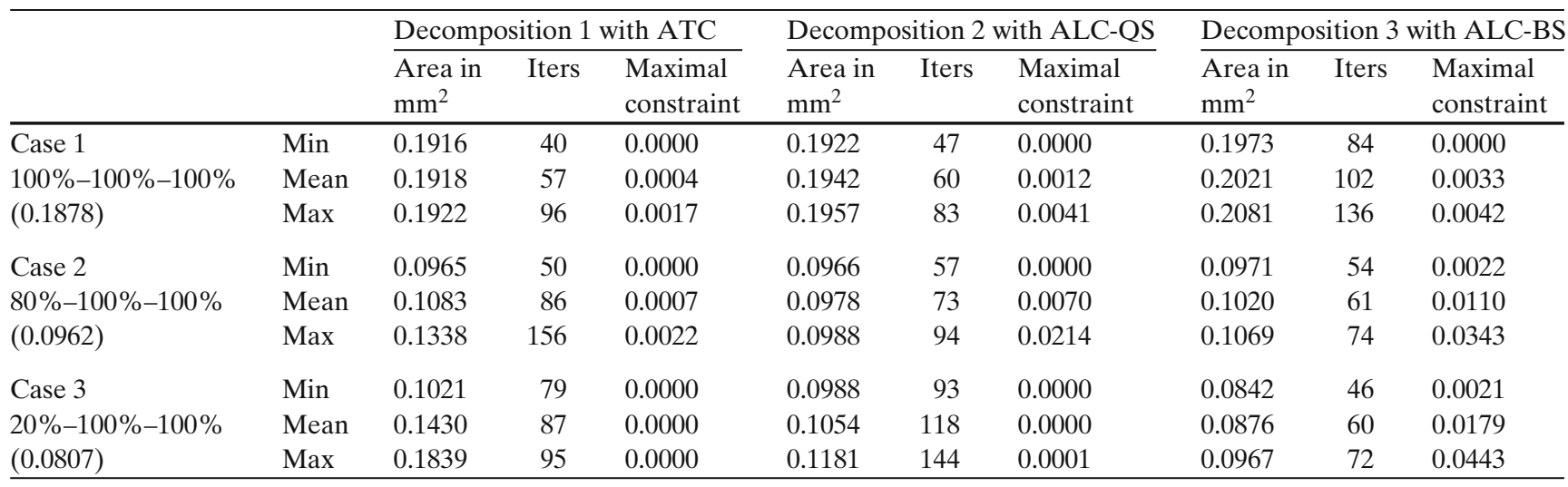

For each design case, the percentage of converged starting points is indicated. Values between parenthesis are optimal areas in mm ${ }^{2}$ obtained with the all-in-one formulation

weight update approach of Tosserams et al. (2008) with $\beta=1.1$ and $\gamma=0.9$ was used. Although no detailed fine-tuning of these parameters has been performed, they appear to work well in practice.

The optimization subproblems were solved with fmincon using default settings. Computational difficulties as observed for the all-in-one formulation are expected at the subproblems as well. To make sure that each subproblem communicated a correct solution, only solutions from a successful fmincon run were used. If a subproblem did not converge, its optimization was restarted from a perturbed starting point. Note that these restarts are infrequent and appear only in the first few iterations. Since augmented Lagrangian coordination requires continuous design variables, Case 4 was not included in the distributed experiments. An alternative coordination approach that handles integer variables can be found in Michalek and Papalambros (2006).

\subsubsection{Decomposition 1 with ATC}

We first consider problem decomposition 1 shown in Fig. 9a. This object-based decomposition has a hierarchical structure, and its solution can be coordinated with the alternating direction method of multipliers for analytical target cascading, denoted here by ATC (Tosserams et al. 2006). ${ }^{2}$ Since ATC allows targets and responses only between two consecutive levels, dummy target-response couples are introduced at the Dynamics subsystem for $S_{\mathrm{d}}, V_{s 0}$, and $C_{\mathrm{p}}$.

The results for the experiments are depicted in Table 5. The results show that ATC is able to find optimal solutions close to those obtained with the

\footnotetext{
${ }^{2}$ ATC is a subclass of ALC, see Tosserams et al. (2008).
}

all-in-one approach for the first two cases. The final case shows less optimistic results since only a few experiments converged to a solution. The remaining starting points either reached the maximum number of iterations (500), or converged to an inconsistent solution. The solutions of Case 3 that did converge showed a relatively high objective function value. Why ATC performs so poorly for this particular case is not clear, but possible explanations may be the non-convexity of the problem, and the lack of global optimality at subproblems due to the local search algorithm.

The average coordination costs for ATC range from around 20 to 100 subproblem optimizations. As can be expected, the average number of subproblem optimizations increases as the number of design variables increases with each case.

\subsubsection{Decomposition 2 with ALC-QS}

The second problem decomposition, shown in Fig. 9b, has only linking variables, and was solved with the augmented Lagrangian coordination approach for quasiseparable problems denoted ALC-QS (Tosserams et al. 2007). The results of Table 5 again show that the distributed approach is able to find solutions similar to the all-in-one solutions for the first two cases, even though only a local search algorithm is used. Case 3 shows a relatively larger difference with the all-inone solution, possibly caused by the non-convexity of the problem. The convergence difficulties for this case associated with ATC were not encountered for this decomposition. The average coordination costs range from around 60 to 120 subproblem optimizations, which is similar to decomposed problem 1. Again, the general trend is that the number of subproblem optimizations increases as the number of design variables increases. 


\subsubsection{Decomposition 3 with ALC-BS}

To coordinate the third problem decomposition of Fig. 9c, we use the ALC variant for block-dependent constraints denoted ALC-BS (Tosserams et al. 2009a) which introduces a master problem to coordinate the coupling between the subsystems. The results for this decomposition are depicted in Table 5. The obtained solutions for all three cases are close to the all-inone results. The average coordination cost is around 60 subproblem optimizations for all but Case 1, which requires an average of 102 subproblem optimizations. In contrast to the previous two problem decompositions, no increasing trend in computational costs can be observed for this decomposition.

\subsubsection{Decomposition 4 with ALC-D}

To coordinate the coupling through the linking variables as well as the coupling constraints for this fourth problem decomposition (Fig. 9d), we use the distributed variant of the augmented Lagrangian coordination approach which we denote by ALC-D (Tosserams et al. 2008). Linking variables are coordinated directly between subsystems, and no coordinating master problem is introduced. We use an inexact inner loop, which is required for the convergence proofs for ALC-D with non-separable constraints. For the penalty parameter updates, we take $\beta=2.0$ and $\gamma=0.5$. We also test an alternating direction approach for which no convergence proof is available with $\beta=1.1$ and $\gamma=$ 0.9. Such an approach with a single iteration for each inner loop has proven to be very efficient for other augmented Lagrangian coordination algorithms. With these additional experiments we investigate whether such an approach is still useful in practice, although no convergence proof is available.

The results presented in Table 6 show that the inexact version of ALC-D are able to find accurate solutions for all cases. The alternating direction approach finds optimal designs for the first two cases, while the solutions for Case 3 are less accurate on average. The solutions costs for the alternating direction version are for some cases only a third of those for the inexact version. The large difference once again illustrates the computational advantages that can be gained by using an alternating direction approach, provided that the obtained solutions are accurate enough.

\subsubsection{General observations for ALC}

In general, the solutions obtained with ALC algorithms for Cases 1 and 2 are all close to those obtained with the all-in-one experiments. The solutions for Case 3 show larger differences. What causes these differences is unknown, but apparently ALC finds these cases harder to coordinate, possibly due to the non-convexities.

The number of converged solutions is much higher for the distributed optimization experiments than for the all-in-one experiments. Where for all-in-one on average only 2 out of the 10 runs converged to a solution, the ALC runs converged from almost all starting points. The most likely explanation for these differences is the fact that subproblems for ALC are restarted from a perturbed starting point when their solution did not converge. Although infrequent in practice, such restarts are mainly observed for the first iteration suggesting that poor initial designs are simply replaced by new

Table 6 Results for Decomposition 4 using ALC-D with an alternating direction and an inexact inner loop

\begin{tabular}{|c|c|c|c|c|c|c|c|}
\hline & & \multicolumn{6}{|c|}{ Decomposition 4 with ALC-D } \\
\hline & & \multicolumn{3}{|c|}{ Alternating direction } & \multicolumn{3}{|c|}{ Inexact inner loop } \\
\hline & & $\begin{array}{l}\text { Area in } \\
\mathrm{mm}^{2}\end{array}$ & Iters & $\begin{array}{l}\text { Maximal } \\
\text { constraint }\end{array}$ & $\begin{array}{l}\text { Area in } \\
\mathrm{mm}^{2}\end{array}$ & Iters & $\begin{array}{l}\text { Maximal } \\
\text { constraint }\end{array}$ \\
\hline$\overline{\text { Case } 1}$ & Min & 0.1918 & 71 & 0.0000 & 0.1916 & 91 & 0.0000 \\
\hline $100 \%-100 \%$ & Mean & 0.1929 & 84 & 0.0042 & 0.1922 & 197 & 0.0016 \\
\hline$(0.1878)$ & Max & 0.1944 & 115 & 0.0118 & 0.1938 & 401 & 0.0122 \\
\hline Case 2 & Min & 0.0971 & 68 & 0.0001 & 0.0972 & 183 & 0.0000 \\
\hline $100 \%-90 \%$ & Mean & 0.0997 & 79 & 0.0032 & 0.1000 & 322 & 0.0005 \\
\hline$(0.0962)$ & Max & 0.1051 & 92 & 0.0098 & 0.1067 & 645 & 0.0020 \\
\hline Case 3 & Min & 0.0860 & 69 & 0.0001 & 0.0827 & 185 & 0.0000 \\
\hline $100 \%-60 \%$ & Mean & 0.1147 & 87 & 0.0028 & 0.0990 & 349 & 0.0027 \\
\hline$(0.0807)$ & Max & 0.3002 & 103 & 0.0101 & 0.1482 & 659 & 0.0131 \\
\hline
\end{tabular}

For each case the percentage of converged starting points is indicated. Values between parenthesis are optimal areas in mm ${ }^{2} \mathrm{obtained}$ with the all-in-one formulation 
ones. The all-in-one implementation does not have such a repairing ability, resulting in a smaller number of converged runs.

The differences between problem decompositions themselves are relatively small. The obtained solutions are very similar, as well as the number of converged solutions. Only Case 3 for the first problem decomposition shows a significantly lower number of converged runs. The coordination costs are similar for all decompositions, which is a little surprising, since the number of linking variables and linking functions is different for each decomposition. However, the results do not show variations in computational costs.

\section{Conclusions and discussion}

This article presents a new multidisciplinary design optimization (MDO) benchmark problem based on the design of a micro-accelerometer. Analysis models describe relevant aspects from mechanics, electrostatics, dynamics, and electronics. The analysis models are analytical, and therefore suitable for numerical optimization algorithms. An area minimization problem is defined to illustrate how these models can be used for optimization purposes. Four design cases are formulated, each with a different number of design variables. Benchmark solutions are derived using a multi-start sequential quadratic programming algorithm.

To illustrate how the models can be used for testing MDO coordination algorithms, four different problem decompositions are presented. Reference results for a number of augmented Lagrangian coordination algorithms are given, and show that these approaches are able to find (near-)optimal designs by performing only local optimization at the individual subsystems. Improvements are expected if a global search is performed at the subproblems.

What makes this problem suitable for benchmark purposes is its fully analytical and therefore reproducible analysis models, the possibility to choose between different design cases, each with a different number of design variables ranging from seven continuous variables to twenty continuous and two discrete variables. Furthermore, the problem is based on an existing device, and can therefore be augmented with more detailed, numerical modeling techniques. Such extensions would improve the accuracy of the analysis, but would also introduce additional practical aspects encountered in MDO such as numerical noise, heterogenous computing environments, and large differences between solution times of subsystems.
The results for the numerical experiments indicate that solving the problem in an all-in-one fashion with a multistart algorithm is more efficient than the reference distributed optimization approach ALC. Since coordination cost reduction is desired for any distributed optimization approach, researchers can use the problem and the all-in-one reference results as a benchmark in their research efforts to reduce the coordination cost associated with distributed optimization. Directions for improvements can be the use of different coordination or subproblem optimization algorithms, the use of surrogate modeling techniques, or reformulating or transforming the problem.

Besides the area minimization problems posed in this article, other design problems such as sensitivity maximization or noise minimization can easily be set up. These alternative problem formulations use the same models but may behave very different from a numerical optimization and coordination perspective.

Acknowledgements The authors would like to thank Albert Groenwold and Chris Dryer of the University of Stellenbosch, and Jaap Flokstra, Reinder Cuperus, and Remco Wiegerink of the University of Twente for their valuable suggestions.

Open Access This article is distributed under the terms of the Creative Commons Attribution Noncommercial License which permits any noncommercial use, distribution, and reproduction in any medium, provided the original author(s) and source are credited.

\section{Appendix: U-spring stiffness matrix analysis}

We begin the analysis of the stiffness of a single Uspring of Fig. 4a by constructing its assembled stiffness matrix. This assembled stiffness $\mathbf{K}_{\text {ass }}$ matrix is constructed from the local stiffness matrices of each beam. The local stiffness matrix $\mathbf{K}_{\text {local }}$ relates the forces $\mathbf{f}=\left[X_{i}, Y_{i}, M_{i}, X_{j}, Y_{j}, M_{j}\right]$ to the displacements $\mathbf{u}=$ $\left[u_{i}, v_{i}, \varphi_{i}, u_{j}, v_{j}, \varphi_{j}\right]$ through $\mathbf{f}=\mathbf{K}_{\text {local }} \mathbf{u}$, and is given by

$$
\mathbf{K}_{\text {local }}=\left[\begin{array}{cccccc}
\frac{E A}{L} & 0 & 0 & -\frac{E A}{L} & 0 & 0 \\
0 & \frac{12 E I}{L^{3}} & \frac{6 E I}{L^{2}} & 0 & -\frac{12 E I}{L^{3}} & \frac{6 E I}{L^{2}} \\
0 & \frac{6 E I}{L^{2}} & \frac{4 E I}{L} & 0 & -\frac{6 E I}{L^{2}} & \frac{2 E I}{L} \\
-\frac{E A}{L} & 0 & 0 & \frac{E A}{L} & 0 & 0 \\
0 & -\frac{12 E I}{L^{3}} & -\frac{6 E I}{L^{2}} & 0 & \frac{12 E I}{L^{3}} & -\frac{6 E I}{L^{2}} \\
0 & \frac{6 E I}{L^{2}} & \frac{2 E I}{L} & 0 & -\frac{6 E I}{L^{2}} & \frac{4 E I}{L}
\end{array}\right]
$$

where $E=160 \cdot 10^{9} \mathrm{~N} / \mathrm{m}^{2}$ is the modulus of elasticity of polysilicon, $A=h w$ is the cross section of the beam, $I=h w^{3} / 12$ is the second moment of inertia of the beam, and $L$ is the beam length. See Fig. $4 \mathrm{~b}$ for a definition of the forces and displacements. For proper 
scaling of the elements of $\mathbf{K}_{\text {local }}$, we take $\mu \mathrm{m}$ as units for all length-related quantities in (79) instead of the more conventional meters.

To create the assembled stiffness matrix, the element stiffness matrices have to be rotated from the local to the global coordinate system. To this end, a rotation matrix $\mathbf{R}=\mathbf{R}(\alpha)$ is used such that

$$
\mathbf{K}_{\text {global }}=\mathbf{R}^{T} \mathbf{K}_{\text {local }} \mathbf{R}
$$

where the matrix $\mathbf{R}=\mathbf{R}(\alpha)$ is given by

$$
\mathbf{R}=\mathbf{R}(\alpha)=\left[\begin{array}{cccccc}
\cos (\alpha) & \sin (\alpha) & 0 & 0 & 0 & 0 \\
-\sin (\alpha) & \cos (\alpha) & 0 & 0 & 0 & 0 \\
0 & 0 & 1 & 0 & 0 & 0 \\
0 & 0 & 0 & \cos (\alpha) & \sin (\alpha) & 0 \\
0 & 0 & 0 & -\sin (\alpha) & \cos (\alpha) & 0 \\
0 & 0 & 0 & 0 & 0 & 1
\end{array}\right]
$$

The three beams and their rotation angles $\alpha$ (the angle from the global $x$ axis to the local $x$ axis) are defined in Table 7 . The numbering of nodes may appear cumbersome, but allows for more convenient notation. Note that the spring softening contribution in the $x$ direction due to electrostatic forces is addressed at the electrostatics subsystem.

To determine the assembled stiffness matrix $\mathbf{K}_{\text {ass }}$ for the whole structure, the local nodes are mapped to the global nodes, and the rotated local stiffness matrices are inserted at the appropriate places of the assembled stiffness matrix $\mathbf{K}_{\text {ass }}$. To this end, a projection matrix $\mathbf{P}^{[i]}$ for beam $i$ can be defined such that

$\mathbf{K}_{\mathrm{ass}}=\sum_{i=1}^{3}\left(\mathbf{P}^{[i]}\right)^{T} \mathbf{K}_{\text {global }}^{[i]} \mathbf{P}^{[i]}$

For beams 1, 2, and 3 the projection matrices are given by

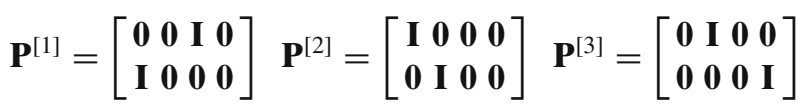

with $\mathbf{I}$ the $3 \times 3$ identity matrix and $\mathbf{0}$ a $3 \times 3$ zero matrix.

Table 7 Beam definitions and rotation angles

\begin{tabular}{llllll}
\hline Beam & $i$ & $j$ & $\alpha$ & Length $L$ & Width $w$ \\
\hline 1 & 3 & 1 & $-\pi / 2$ & $l_{\mathrm{b} 1}$ & $w_{\mathrm{b}}$ \\
2 & 1 & 2 & $\pi$ & $l_{\mathrm{b} 2}$ & $w_{\mathrm{b} 2}$ \\
3 & 2 & 4 & $\pi / 2$ & $l_{\mathrm{b} 3}$ & $w_{\mathrm{b}}$ \\
\hline
\end{tabular}

\section{Computation of reaction forces}

With the assembled stiffness matrix $\mathbf{K}_{\text {ass }}$ defined, we are now ready to derive the mechanical stiffness.

First, we consider the boundary conditions of the spring. From the boundary conditions we know that node 3 is clamped $\left[u_{3}, v_{3}, \varphi_{3}\right]=[0,0,0]$, node 4 is subjected to forced displacements $\mathbf{u}_{4}=[u, v, \varphi]$, and the reaction forces at nodes 1 and 2 are zero: $\left[X_{1}, Y_{1}, Z_{1}\right]=\left[X_{2}, Y_{2}, Z_{2}\right]=[0,0,0]$. The displacements $\left[u_{1}, v_{1}, \theta_{1}\right]$ and $\left[u_{2}, v_{2}, \varphi_{2}\right]$, and reaction forces at node $3\left[X_{3}, Y_{3}, M_{3}\right]$ and node $4\left[F_{4}, F_{4}, M_{4}\right]$ are unknown.

To compute the unknown displacements and forces, the displacement and force vectors are split up into two parts: a known part (boundary conditions) and an unknown part (free nodes), such that $\mathbf{u}_{\mathrm{ass}}=\left[\mathbf{u}_{\text {free }}^{T}, \mathbf{u}_{\mathrm{bc}}^{T}\right]^{T}$ and $\quad \mathbf{f}_{\mathrm{ass}}=\left[\mathbf{f}_{\mathrm{bc}}^{T}, \mathbf{f}_{\text {free }}^{T}\right]^{T}$, where $\quad \mathbf{u}_{\text {free }}=\left[u_{1}, v_{1}, \varphi_{1}, u_{2}\right.$, $\left.v_{2}, \varphi_{2}\right]^{T}, \mathbf{u}_{\mathrm{bc}}=\left[u_{3}, v_{3}, \varphi_{3}, u_{4}, v_{4}, \varphi_{4}\right]=[0,0,0, u, v, \varphi]^{T}$, $\mathbf{f}_{\mathrm{bc}}=\left[X_{1}, Y_{1}, Z_{1}, X_{2}, Y_{2}, Z_{2}\right]=[0,0,0,0,0,0]^{T}$, and $\mathbf{f}_{\text {free }}=\left[X_{3}, Y_{3}, M_{3}, X_{4}, Y_{4}, M_{4}\right]^{T}$.

Under these conventions, the unknowns can be determined from the system of equations $\mathbf{K}_{\text {ass }} \mathbf{u}_{\text {ass }}=\mathbf{f}_{\text {ass }}$, which is given by

$$
\left[\begin{array}{ll}
\mathbf{K}_{11} & \mathbf{K}_{12} \\
\mathbf{K}_{12}^{T} & \mathbf{K}_{22}
\end{array}\right]\left[\begin{array}{c}
\mathbf{u}_{\text {free }} \\
\mathbf{u}_{\mathrm{bc}}
\end{array}\right]=\left[\begin{array}{c}
\mathbf{f}_{\mathrm{bc}} \\
\mathbf{f}_{\text {free }}
\end{array}\right]
$$

where $\mathbf{K}_{11}, \mathbf{K}_{12}$, and $\mathbf{K}_{22}$ are $6 \times 6$ submatrices of the assembled stiffness matrix $\mathbf{K}_{\text {ass }}$.

From this system, and because $\mathbf{f}_{\mathrm{bc}}=0$, the free displacements are given by

$\mathbf{u}_{\text {free }}=\mathbf{K}_{11}^{-1}\left(\mathbf{f}_{\mathrm{bc}}-\mathbf{K}_{12} \mathbf{u}_{\mathrm{bc}}\right)=-\mathbf{K}_{11}^{-1} \mathbf{K}_{12} \mathbf{u}_{\mathrm{bc}}$

The unknown reaction forces $\mathbf{f}_{\text {free }}=\left[X_{3}, Y_{3}, Z_{3}\right.$, $\left.X_{4}, Y_{4}, Z_{4}\right]^{T}$ are given by

$$
\begin{aligned}
\mathbf{f}_{\text {free }} & =\mathbf{K}_{12}^{T} \mathbf{u}_{\text {free }}+\mathbf{K}_{22} \mathbf{u}_{\mathrm{bc}} \\
& =\left(\mathbf{K}_{22}-\mathbf{K}_{12}^{T} \mathbf{K}_{11}^{-1} \mathbf{K}_{12}\right) \mathbf{u}_{\mathrm{bc}}=\tilde{\mathbf{K}} \mathbf{u}_{\mathrm{bc}}
\end{aligned}
$$

where $\tilde{\mathbf{K}}=\mathbf{K}_{22}-\mathbf{K}_{12}^{T} \mathbf{K}_{11}^{-1} \mathbf{K}_{12}$.

Let $\tilde{\mathbf{K}}_{33}, \tilde{\mathbf{K}}_{34}$, and $\tilde{\mathbf{K}}_{44}$ be $3 \times 3$ submatrices of $\tilde{\mathbf{K}}$ such that

$\tilde{\mathbf{K}}=\left[\begin{array}{ll}\tilde{\mathbf{K}}_{33} & \tilde{\mathbf{K}}_{34} \\ \tilde{\mathbf{K}}_{34}^{T} & \tilde{\mathbf{K}}_{44}\end{array}\right]$

Then since $\mathbf{u}_{\mathrm{bc}}=[0,0,0, u, v, \varphi]$, the reaction forces at node 4 can be determined by

$$
\left[\begin{array}{c}
X_{4} \\
Y_{4} \\
M_{4}
\end{array}\right]=\tilde{\mathbf{K}}_{44}\left[\begin{array}{c}
u \\
v \\
\varphi
\end{array}\right]=\left[\begin{array}{ccc}
K_{\mathrm{x}} & K_{\mathrm{xy}} & K_{\mathrm{x} \varphi} \\
K_{\mathrm{xy}} & K_{\mathrm{y}} & K_{\mathrm{y} \varphi} \\
K_{\mathrm{x} \varphi} & K_{\mathrm{y} \varphi} & K_{\varphi}
\end{array}\right]\left[\begin{array}{c}
u \\
v \\
\varphi
\end{array}\right]
$$

The elements of $\tilde{\mathbf{K}}_{44}$ can at this point be un-scaled to SI units again. 
For a perfectly symmetrical structure the cross-axis stiffness $K_{\mathrm{xy}}=0$. In practice, small variations of dimensions due to manufacturing introduce a non-zero stiffness $K_{\mathrm{xy}}$ that initiate deflections in the $x$ direction due to accelerations in the $y$ direction. For our analysis, we will assume $K_{\mathrm{xy}}=0$.

\section{References}

Agte JS, Sobieszczanski-Sobieski J, Sandusky RR Jr (1999) Supersonic business jet design through bi-level integrated system synthesis. In: Proceedings of the world aviation conference, San Fransisco, CA, MCB Press, SAE paper 1999$01-5622$

Alexandrov NM, Lewis RM (1999) Comparative properties of collaborative optimization and other approaches to MDO. In: ASMO UK/ISSMO conference on engineering design optimization. MCB University Press, Bradford, pp 39-46

Analog Devices Inc (1998) Adxl150/adxl250 data sheet

Balling RJ, Sobieszczanski-Sobieski J (1996) Optimization of coupled systems: a critical overview of approaches. AIAA J 34(1):6-17

Balling RJ, Wilkinson CA (1997) Execution of multidisciplinary design optimization approaches on common test problems. AIAA J 35(1):178-186

Boser BE, Howe RT (1995) Surface micromachined accelerometers. In: Proceedings of the IEEE custom integrated circuit conference, pp 337-344

Braun RD (1996) Collaborative optimization: an architecture for large-scale distributed design. PhD thesis, Stanford University

Cho Y, Pisano AP, Howe RT (1994) Viscous damping model for laterally oscillating microstructures. Journal of Microelectromechanical Systems 3(2):81-87

Cramer EJ, Dennis JE, Frank PD, Lewis RM, Shubin GR (1994) Problem formulation for multidisciplinary optimization. SIAM J Optim 4(4):754-776

DeMiguel AV, Murray W (2006) A local convergence analysis of bilevel decomposition algorithms. Optimization and Engineering 7(2):99-133

Haftka RT, Watson LT (2005) Multidisciplinary design optimization with quasiseparable subsystems. Optimization and Engineering 6(1):9-20

Leondes CT (2006) MEMS/NEMS handbook: techniques and applications. Design methods in MEMS/NEMS, vol 1. Springer, New York

Mathworks (2008) Matlab user guide, version 7. Mathworks, Natick

Michalek J, Papalambros PY (2006) BB-ATC: analytical target cascading using branch and bound for mixed integer nonlinear programming. In: Proceedings of the ASME design engineering technical conferences, Philadelphia, PA

Michelena N, Kim HM, Papalambros PY (1999) A system partitioning and optimization approach to target cascading. In: Proceedings of the 12th international conference on engineering design, Munich, Germany

Mukherjee T (2003) MEMS design and verification. In: Proceedings of the IEEE international test conference, Charlotte, $\mathrm{NC}$

Mukherjee T, Iyer S, Fedder GK (1998) Optimization-based synthesis of microresonators. Sens Actuators A Phys 70(1-2):118-127
Mukherjee T, Zhou Y, Fedder GK (1999) Automated optimal synthesis of microaccelerometers. In: Proceeding of the 12th annual IEEE international micro electro mechanical system conference, Orlando, FL, pp 326-331

Padula SL, Alexandrov NM, Green LL (1996) MDO test suite at nasa langley research center. In: Proceedings of the 6th AIAA/USAF/NASA/ISSMO multidisciplinary analysis and optimization conference, Bellevue, WA. AIAA paper 19964028

Papila M, Haftka RT, Nishida T, Sheplak M (2006) Piezoresistive microphone design pareto optimization: tradeoff between sensitivity and noise floor. Journal of Microelectromechanical Systems 15(6):1632-1643

Pedersen CBW, Seshia AA (2004) On the optimization of compliant force amplifier mechanisms for surface micromachined resonant accelerometers. J Micromechanics Microengineering 14:1281-1293

Perez RE, Liu HHT, Behdinan K (2004) Evaluation of multidisciplinary optimization approaches for aircraft conceptual design. In: Proceedings of the 10th AIAA/ISSMO multidisciplinary analysis and optimization conference, Albany, NY. AIAA paper 2004-4537

Samuels H (1996) Single- and dual-axis micromachined accelerometers adxl150 \& adxl250: new complete low-noise 50-g accelerometers. Analog Dialogue 30(4)

Senturia SF (2001) Microsystem design. Springer

Sobieszczanski-Sobieski J, James BB, Dovi AR (1983) Structural optimization by multilevel decomposition. AIAA paper 1983-0832

Sobieszczanski-Sobieski J, Altus TD, Phillips M, Sandusky RR $\mathrm{Jr}$ (2003) Bilevel integrated system synthesis for concurrent and distributed processing. AIAA J 41(10):1996-2003

Tosserams S, Etman LFP, Papalambros PY, Rooda JE (2006) An augmented Lagrangian relaxation for analytical target cascading using the alternating direction method of multipliers. Struct Multidiscipl Optim 31(3):176-189

Tosserams S, Etman LFP, Rooda JE (2007) An augmented Lagrangian decomposition method for quasi-separable problems in MDO. Struct Multidiscipl Optim 34(3):211227

Tosserams S, Etman LFP, Rooda JE (2008) Augmented Lagrangian coordination for distributed optimal design in MDO. Int J Numer Methods Eng 73(13):1885-1910

Tosserams S, Etman LFP, Rooda JE (2009a) Block-separable linking constraints in augmented Lagrangian coordination. Struct Multidiscipl Optim 37(5):521-527

Tosserams S, Etman LFP, Rooda JE (2009b) A classification of methods for distributed system optimization based on formulation structure. Struct Multidiscipl Optim. doi:10. 1007/s00158-008-0347-z

University of Buffalo (2009) Multidisciplinary optimization and engineering laboratory. http://www.eng.buffalo.edu/ Research/MODEL/. Accessed 25 March 2009

Veijola T, Pursula A, Råback P (2005) Extending the validity of squeezed-film damper models with elongations of surface dimensions. J Micromechanics Microengineering 15:16241636

Yi SI, Shin MK, Park GJ (2008) Comparison of MDO methods with mathematical examples. Struct Multidiscipl Optim 35(5):391-402

Yuan W, Chang H, Li W, Ma B (2006) Application of an optimization methodology for multidisciplinary system design of microgyroscopes. Microsyst Technol 12(4):315323

Zhou Y (1998) Layout synthesis of accelerometers. Master's thesis, Carnegie Mellon University 